\title{
The evaluation of monoclonal gammopathy of renal significance: a consensus report of the International Kidney and Monoclonal Gammopathy Research Group
}

Nelson Leung $\mathbb{D}^{1 *}$, Frank Bridoux ${ }^{2}$, Vecihi Batuman ${ }^{3}$, Aristeidis Chaidos ${ }^{4}$, Paul Cockwell ${ }^{5}$, Vivette D. D'Agati ${ }^{6}$, Angela Dispenzieri' ${ }^{1}$ Fernando C. Fervenza ${ }^{1}$, Jean-Paul Fermand ${ }^{7}$, Simon Gibbs ${ }^{8}$, Julian D. Gillmore ${ }^{9}$, Guillermo A. Herrera ${ }^{10}$, Arnaud Jaccard ${ }^{11}$, Dragan Jevremovic ${ }^{1}$, Efstathios Kastritis ${ }^{12}$, Vishal Kukreti ${ }^{13}$, Robert A. Kyle ${ }^{1}$, Helen J. Lachmann ${ }^{9}$, Christopher P. Larsen ${ }^{14}$, Heinz Ludwig ${ }^{15}$, Glen S. Markowitz ${ }^{6}$, Giampaolo Merlini ${ }^{16}$, Peter Mollee ${ }^{17}$, Maria M. Picken ${ }^{18}$, Vincent S. Rajkumar' ${ }^{1}$, Virginie Royal ${ }^{19}$, Paul W. Sanders (iD ${ }^{20}$, Sanjeev Sethi ${ }^{1}{ }^{1}$, Christopher P. Venner ${ }^{21}$, Peter M. Voorhees ${ }^{22}$, Ashutosh D. Wechalekar ${ }^{9}$, Brendan M. Weiss ${ }^{23}$ and Samih H. Nasr ${ }^{1}$

Abstract | The term monoclonal gammopathy of renal significance (MGRS) was introduced by the International Kidney and Monoclonal Gammopathy Research Group (IKMG) in 2012. The IKMG met in April 2017 to refine the definition of MGRS and to update the diagnostic criteria for MGRS-related diseases. Accordingly, in this Expert Consensus Document, the IKMG redefines MGRS as a clonal proliferative disorder that produces a nephrotoxic monoclonal immunoglobulin and does not meet previously defined haematological criteria for treatment of a specific malignancy. The diagnosis of MGRS-related disease is established by kidney biopsy and immunofluorescence studies to identify the monotypic immunoglobulin deposits (although these deposits are minimal in patients with either C3 glomerulopathy or thrombotic microangiopathy). Accordingly, the IKMG recommends a kidney biopsy in patients suspected of having MGRS to maximize the chance of correct diagnosis. Serum and urine protein electrophoresis and immunofixation, as well as analyses of serum free light chains, should also be performed to identify the monoclonal immunoglobulin, which helps to establish the diagnosis of MGRS and might also be useful for assessing responses to treatment. Finally, bone marrow aspiration and biopsy should be conducted to identify the lymphoproliferative clone. Flow cytometry can be helpful in identifying small clones. Additional genetic tests and fluorescent in situ hybridization studies are helpful for clonal identification and for generating treatment recommendations. Treatment of MGRS was not addressed at the 2017 IKMG meeting; consequently, this Expert Consensus Document does not include any recommendations for the treatment of patients with MGRS.

*e-mail: leung.nelson@ mayo.edu
The concept of monoclonal gammopathy of undetermined significance (MGUS) was first introduced in 1978 by Robert Kyle ${ }^{1}$. This premalignant condition is characterized by the presence of a serum monoclonal immunoglobulin $<30 \mathrm{~g} / \mathrm{l}$ and $<10 \%$ monoclonal bone marrow plasma cells in a patient who does not have any organ damage attributable to the monoclonal immunoglobulin. Conversion of MGUS to malignancy, which mandates the initiation of appropriate treatment, is indicated by the development of disease-specific features. For example, conversion to multiple myeloma (MM) is indicated by the occurrence of one or more myeloma-defining events, such as hypercalcaemia, renal impairment, anaemia, lytic bone lesions or an event suggestive of impending myeloma (such as a serum involved:uninvolved free light-chain ratio $>100,>60 \%$ bone marrow plasma cells or $\geq 1$ bone lesions on MRI $)^{2}$. Progression to Waldenström macroglobulinaemia (WM) is indicated by the development of anaemia, thrombocytopenia, bulky adenopathy or organomegaly, blood hyperviscosity, severe neuropathy, amyloidosis, cryoglobulinaemia, cold agglutinin disease or malignant transformation ${ }^{3}$. Similarly, treatment for chronic lymphocytic leukaemia (CLL) is initiated when a patient with MGUS develops cytopenias, progressive or symptomatic lymphadenopathy, organomegaly or 
Bence Jones protein

Monoclonal immunoglobulin light chains detected in the urine of patients with multiple myeloma and other haematological malignancies. constitutional symptoms ${ }^{4}$. Patients with MGUS who do not yet exhibit any of these disease-specific features do not require treatment but should undergo careful monitoring ${ }^{2,5-9}$.

The kidney is commonly involved in these haematological malignancies. Light-chain cast nephropathy is now considered a myeloma-defining event, although it is not exclusive to $\mathrm{MM}^{10}$. In addition to cryoglobulinaemic glomerulonephritis, a variety of other kidney diseases have been observed in patients with WM, including immunoglobulin light-chain (AL) amyloidosis, monoclonal immunoglobulin deposition disease (MIDD), light-chain proximal tubulopathy (LCPT) and, on rare occasions, cast nephropathy ${ }^{11-13}$. Similar renal lesions have also been described in patients with $\mathrm{CLL}^{14}$. Importantly, however, these kidney diseases have also been described in patients with a low clonal burden (defined as monoclonal immunoglobulin $<30 \mathrm{~g} / 1$ and $<10 \%$ monoclonal bone marrow plasma cells) who therefore do not meet the diagnostic criteria for MM

\footnotetext{
Author addresses

${ }^{1}$ Division of Nephrology, Hematology, Department of Laboratory Medicine and Pathology, Mayo Clinic, Rochester, MN, USA.

2Department of Nephrology, Centre Hospitalier Universitaire et Université de Poitiers, Poitiers, France; CNRS UMR7276, Limoges, France; and Centre de Référence Amylose AL et Autres Maladies par Dépôt d'Immunoglobulines Monoclonales, Poitiers, France.

${ }^{3}$ Veterans Administration Medical Center, New Orleans, LA, USA and Tulane University Medical School, Tulane, LA, USA.

${ }^{4}$ Centre for Haematology, Department of Medicine, Imperial College London and Imperial College Healthcare NHS Trust, Hammersmith Hospital, London, UK.

${ }^{5}$ Department of Nephrology, Renal Medicine - University Hospitals Birmingham NHS

Foundation Trust, Queen Elizabeth Hospital, Birmingham, UK.

${ }^{6}$ Department of Pathology, Renal Pathology Laboratory, Columbia University,

College of Physicians and Surgeons, New York, NY, USA.

${ }^{7}$ Department of Haematology and Immunology, University Hospital St Louis, Paris, France. ${ }^{8}$ The Victorian and Tasmanian Amyloidosis Service, Department of Haematology, Monash Univerity Easter Health Clinical School, Melbourne, Victoria, Australia.

${ }^{9}$ National Amyloidosis Centre, Centre for Amyloidosis and Acute Phase Proteins, Division of Medicine, Royal Free Campus, University College London, London, UK.

${ }^{10}$ Department of Pathology and Translational Pathobiology, Louisiana State University Health Sciences Center, Shreveport, LA, USA.

${ }^{11}$ Service d'Hématologie et de Thérapie Cellulaire, Centre de Référence des Amyloses

Primitives et des Autres Maladies par Dépôts d'Immunoglobuline, CHU Limoges, Limoges, France.

${ }^{12}$ Department of Clinical Therapeutics, School of Medicine National and Kapodistrian University of Athens Alexandra Hospital, Athens, Greece.

${ }^{13}$ University Health Network, Princess Margaret Cancer Centre, Toronto, Canada.

${ }^{14}$ Arkana Laboratories, Little Rock, AR, USA.

${ }^{15}$ Wilhelminen Cancer Research Institute, Wilhelminenspital, Vienna, Austria.

${ }^{16}$ Amyloidosis Research and Treatment Center, IRCCS Policlinico San Matteo, University of Pavia, Pavia, Italy.

${ }^{17}$ Haematology Department, Princess Alexandra Hospital and School of Medicine, University of Queensland, Brisbane, Australia.

${ }^{18}$ Department of Pathology, Loyola University Medical Center, Maywood, IL, USA.

${ }^{19}$ Department of Pathology, Hôpital Maisonneuve-Rosemont, Université de Montreal, Montreal, Quebec, Canada.

${ }^{20}$ Department of Medicine, University of Alabama at Birmingham and Department of Veterans Affairs Medical Center, Birmingham, AL, USA.

${ }^{21}$ Cross Cancer Institute, University of Alberta, Edmonton, Alberta, Canada.

${ }^{22}$ Department of Hematologic Oncology and Blood Disorders, Levine Cancer Institute,

Atrium System, Charlotte, NC, USA.

${ }^{23}$ Abramson Cancer Center, University of Pennsylvania, Perelman School of Medicine, Philadelphia, PA, USA.
}

or other malignancies. In the past, these patients were categorized as having 'idiopathic' light-chain disposition disease or 'primary' amyloidosis ${ }^{15,16}$. The fact that these kidney lesions have been replicated in animal models by Bence Jones protein injections alone further supports the notion that the presence of MM is not required $^{17,18}$. For this reason, the International Myeloma Working Group does not consider patients with plasma cell dyscrasia and kidney diseases other than cast nephropathy to have MM unless they also exhibit other myeloma-defining events ${ }^{2}$.

The occurrence of kidney diseases associated with a monoclonal gammopathy in the absence of symptomatic MM, WM or CLL is increasingly recognized ${ }^{10}$. Most of these patients have a small, low-grade clonal disorder that is similar to MGUS, although (unlike MGUS) these clones do cause vital organ damage - including neuropathy, cardiomyopathy, hepatic dysfunction and dermopathy - mediated by the monoclonal immunoglobulin ${ }^{2,19,20}$. The clonal aetiology of these diseases results in clinical features that differ from those of non-monoclonal gammopathies, such as membranous nephropathy or IgA nephropathy. For example, monoclonal immunoglobulin-related diseases tend to be progressive and are unlikely to undergo spontaneous remission ${ }^{21-25}$. Monoclonal immunoglobulin-related diseases also show higher rates of recurrence after kidney transplantation (often $>80 \%$ ) than their nonmonoclonal counterparts ${ }^{26-28}$. Monoclonal diseases are poorly responsive to conventional immunosuppression and instead require clone-directed therapy ${ }^{25,29-32}$.

Increasing recognition of the relationship between monoclonal gammopathies and kidney disease generated the need for more-accurate classification of these disorders, which were previously often misdiagnosed or categorized as unclassifiable by existing disease criteria. Moreover, as the use of cytotoxic therapy is typically limited to patients with MM, WM or CLL, patients with monoclonal gammopathy-related kidney diseases (who do not meet the criteria for these malignancies) were left without access to these essential drugs ${ }^{2}$. Accordingly, a series of meetings was organized by the International Kidney and Monoclonal Gammopathy Research Group (IKMG) with the aim of designating these clonal disorders as pathologies distinct from MGUS and thereby enabling government agencies to allocate resources for their treatment. In 2012, the IKMG introduced the term monoclonal gammopathy of renal significance (MGRS) to describe haematological conditions that produce a monoclonal immunoglobulin associated with kidney injury $^{33}$. Since then, the IKMG has published recommendations for the treatment of $\mathrm{MGRS}^{34}$ and a classification scheme for MGRS-related renal lesions ${ }^{35}$. The IKMG met again in New Orleans, Louisiana, United States, in 2017 to update the classification of MGRS-associated renal lesions as well as to refine the definition of MGRS. The present Expert Consensus Document is derived from these discussions, which occurred both face to face and in e-mail exchanges that incorporated the views of IKMG members who could not be present. The treatment of MGRS was not discussed at the meeting; therefore, this topic is not updated in this consensus document. 


\section{Box 1 | Updated definition of MGRS}

The following consensus view of monoclonal gammopathy of renal significance (MGRS) has emerged. The term MGRS applies specifically to any B cell or plasma cell clonal lymphoproliferation with both of the following characteristics:

- One or more kidney lesions that are related to the produced monoclonal immunoglobulin

- The underlying B cell or plasma cell clone does not cause tumour complications or meet any current haematological criteria for specific therapy

\section{Updated definition of MGRS}

The original definition of MGRS included all small $B$ cell clones that produced a toxic monoclonal protein ${ }^{33}$. Although this definition was based on the dangerous small B cell clones concept ${ }^{19}$, the nature of the clonal disease was not well defined. Specific questions arose regarding whether patients with smouldering (indolent) MM (SMM) or smouldering (indolent) WM (SWM) should be considered to have MGRS. Similar confusion existed with regard to the inclusion of patients with low-grade CLL or lymphoma, who do have a diagnosis of a malignancy but do not require treatment. The new definition includes all B cell and plasma cell clonal proliferative disorders that do not require immediate treatment for the clonal disease. In addition, the toxic monoclonal protein is now specified to be a nephrotoxic monoclonal immunoglobulin ${ }^{33}$.

The new IKMG consensus definition of MGRS (BOX 1) includes all B cell or plasma cell proliferative disorders (such as SMM, SWM and monoclonal B cell lymphocytosis (MBL; a diagnosis that is the equivalent of MGUS for clones of the CLL lineage)) that produce a nephrotoxic monoclonal immunoglobulin ${ }^{1,4,36,37}$. Low-grade CLL and low-grade B cell non-Hodgkin lymphomas, such as marginal zone lymphoma, mantle-cell lymphoma or mucosa-associated lymphoid tissue (MALT) lymphoma are also considered to be MGRS when they are associated with renal lesions ${ }^{38-41}$ (TABLE 1). These low-grade proliferative disorders would be classified as MGUS, and affected patients would be monitored for progression but not offered treatment if not for the renal injury ${ }^{19}$. In patients who develop renal lesions as a result of the monoclonal immunoglobulin, therapeutic intervention is required to prevent further damage resulting in end-stage renal disease. Accordingly, the diagnosis of MGRS does not require the presence of any defining features of an overt lymphoplasmacytic malignancy and particularly not the presence of any myeloma-defining event.

Once the haematological condition progresses to overt MM, WM, advanced stage CLL or malignant lymphoma (as defined by their respective established disease criteria), these diseases are no longer considered MGRS and affected patients are managed according to disease-specific protocols.

Size is not everything small B cell-derived clones

might synthesize a very toxic monoclonal immunoglobulin that produces devastating systemic damage and a progressive or even fatal clinical course. to as MGRS-associated lesions, conditions or disorders. Thus, for instance, classic AL amyloidosis might be considered an MGRS-associated condition when renal involvement is present. By contrast, the term $\mathrm{MM}$-associated AL amyloidosis would be used when the same renal condition is associated with a symptomatic high tumour mass accompanied by at least one classic myeloma-defining event.

The type of renal lesion is governed by the innate structural characteristics and physicochemical properties of the monoclonal immunoglobulin rather than by the features of the clone that produced $\mathrm{it}^{17}$. Except for C3 glomerulopathy and thrombotic microangiopathy, which are not associated with renal deposition of monoclonal immunoglobulin, most MGRS-associated lesions are caused by the deposition of entire or parts of the monoclonal immunoglobulins or of various products of aggregation. Monoclonal immunoglobulin deposits in the kidney are generally restricted to immunoglobulin light chains (except in diseases that show a monoclonal immunoglobulin heavy-chain restriction, such as heavy-chain deposition disease or immunoglobulin heavy-chain amyloidosis). For example, in AL amyloidosis, the renal deposits are composed of only a single light chain ${ }^{35}$. In conditions where the entire immunoglobin is deposited, demonstration of both heavy-chain and light-chain restrictions are required to provide evidence of monoclonality.

The classification scheme proposed in 2017 by the IKMG for MGRS-associated lesions (FIG. 2) is based on the findings of immunofluorescence studies and the ultrastructural appearance of the deposits on electron microscopy. However, electron microscopy is not universally available, even in industrialized countries; consequently, the IKMG classification encourages but does not mandate the use of electron microscopy in the assessment of MGRS-associated disorders. By contrast, light microscopy and immunofluorescence studies with a full panel of antibodies are invariably required. The renal deposits are initially categorized as organized, non-organized and non-immunoglobulin. At the 2017 IKMG meeting in New Orleans, two additional subcategories were added to the non-organized and non-immunoglobulin categories of the classification scheme $\mathrm{e}^{35}$. Thrombotic microangiopathy associated with monoclonal gammopathy was provisionally added as a subcategory of non-immunoglobulin deposits ${ }^{42}$, and a miscellaneous subcategory was added to the non-organized deposit category, which applies to pathological entities that are ultrastructurally similar to a non-monoclonal-immunoglobulin-related disease but are only sometimes associated with a monoclonal gammopathy. The MGRS-associated disorders included in this classification are discussed in more detail below.

Lesions with organized deposits. Organized deposits of monoclonal immunoglobulins can be further divided into fibrillar, microtubular or crystalline and/or inclusionary forms (FIG. 3). Immunoglobulin-related amyloidosis, which includes subtypes with light-chain, heavy-chain and both heavy-and-light-chain deposition 
Table 1 | Characteristics of clonal B cell and plasma cell proliferative disorders

\begin{tabular}{|c|c|c|c|c|c|}
\hline Disease & Clone & $\begin{array}{l}\text { Bone marrow } \\
\text { involvement }\end{array}$ & Immunoglobulin & M-spike & $\begin{array}{l}\text { Organ damage and/or } \\
\text { involvement }\end{array}$ \\
\hline MGUS & Any & $<10 \%$ & Any & $<30 \mathrm{~g} / \mathrm{l}$ & None \\
\hline Smouldering $\mathrm{MM}^{\mathrm{a}}$ & Plasma cell & $10-60 \%$ & Any & $\geq 30 \mathrm{~g} / \mathrm{l}$ & None \\
\hline$M^{a}$ & Plasma cell & $\geq 10 \%$ & Any & $\geq 30 \mathrm{~g} / \mathrm{l}$ & $\begin{array}{l}\text { SLiM CRAB: } 60 \% \text { bone marrow } \\
\text { plasma cells, involved:uninvolved } \\
\text { free light-chain ratio }>100 \text {, } \\
>1 \text { bone lesion on MRI, } \\
\text { hypercalcaemia, renal impairment, } \\
\text { anaemia and lytic bone lesions }\end{array}$ \\
\hline Smouldering $\mathrm{WM}^{\mathrm{a}}$ & $\begin{array}{l}\text { Lymphoplasmacytic lymphoma } \\
\text { clone }^{\text {b }}\end{array}$ & $\geq 10 \%$ & $\lg M$ & $\geq 30 \mathrm{~g} / \mathrm{l}$ & Absent \\
\hline$W^{a}$ & $\begin{array}{l}\text { Lymphoplasmacytic lymphoma } \\
\text { clone }^{\text {b }}\end{array}$ & $\geq 10 \%$ & $\lg M$ & $\geq 30 \mathrm{~g} / \mathrm{l}$ & $\begin{array}{l}\text { Anaemia, hyperviscosity, } \\
\text { constitutional symptoms, } \\
\text { bulky lymphadenopathy, } \\
\text { hepatosplenomegaly and } \\
\text { neuropathy }\end{array}$ \\
\hline MBL & B-cell clone ${ }^{c}$ & $\begin{array}{l}\text { Peripheral } \\
\text { B-cell count } \\
<5 \times 10^{9} /\end{array}$ & Any & Any & $\begin{array}{l}\text { Absence of lymph node } \\
\text { involvement }\end{array}$ \\
\hline CLL & B-cell clone ${ }^{c}$ & $\begin{array}{l}\text { Peripheral } \\
\text { B-cell count } \\
>5 \times 10^{9} / \mathrm{l}\end{array}$ & Any & Any & $\begin{array}{l}\text { Adenopathy, anaemia and } \\
\text { thrombocytopenia }\end{array}$ \\
\hline $\begin{array}{l}\text { Other B cell lympho- } \\
\text { proliferative disorders }\end{array}$ & $\begin{array}{l}\text { Pan B-cell markers } \\
\left(\mathrm{CD} 19^{+} \mathrm{CD} 20^{+} \mathrm{CD} 79^{+} \mathrm{CD} 22^{+} \mathrm{PAX} 5^{+}\right)\end{array}$ & $\begin{array}{l}\text { Presence or } \\
\text { absence }\end{array}$ & Any & Any & Adenopathy and splenomegaly \\
\hline
\end{tabular}

(AL, AH and AHL, respectively), has traditionally been recognized as the only condition in the fibrillar category ${ }^{43}$. However, monoclonal fibrillary glomerulonephritis has occasionally also been reported ${ }^{44}$. Amyloid fibrils stain with Congo red and are solid, non-branching and randomly arranged, with diameters of 7-12 nm (FIG. 3a). Amyloid fibrils involve glomeruli and blood vessels in the vast majority of patients and the interstitium in roughly $60 \%$ of patients (FIG. 2). Intratubular cytoplasmic AL amyloidosis occurs rarely ${ }^{45}$. The randomly arranged fibrils seen in fibrillary glomerulonephritis are on average twice as thick $(10-30 \mathrm{~nm})$ as those observed in amyloidosis (FIG. 3b) and generally do not stain with Congo red ${ }^{44}$. A small subgroup (7-17\%) of patients with fibrillary glomerulonephritis demonstrates clinical evidence of a monoclonal gammopathy. In 3-15\% of these patients, the IgG deposits exhibit light-chain restriction $^{44,46,47}$, and this pathology is termed monoclonal fibrillary glomerulonephritis. Glomerular staining for DnaJ homologue subfamily B member 9 (DNAJB9) is a reliable marker for fibrillary glomerulonephritis ${ }^{48}$. This feature can be used to distinguish monoclonal fibrillary glomerulonephritis from AHL and AH amyloidosis, especially as fibrillary glomerulonephritis can sometimes show Congo red staining ${ }^{49,50}$.

Immunotactoid glomerulonephritis and cryoglobulinaemic glomerulonephritis are the two diseases that feature microtubular immunoglobulin deposits (FIG. 3c). Microtubules can be distinguished from fibrils by their hollow centres and large diameters $(17-52 \mathrm{~nm})^{51}$. Only type I and II cryoglobulinaemias are considered to be
MGRS-associated disorders because type III cryoglobulinaemia is associated solely with polyclonal immunoglobulins. Immunotactoid glomerulonephritis is usually a renal-limited disease, whereas systemic manifestations including vasculitic rashes, peripheral neuropathy and arthralgias are common in patients with cryoglobulinaemia. Moreover, immunotactoid glomerulonephritis is not associated with cryoglobulinaemia and does not display the typical characteristics of cryoglobulinaemic glomerulonephritis (namely, glomerular protein thrombi and arterial or arteriolar vasculitic lesions). The glomerular deposits in immunotactoid glomerulonephritis are uniformly composed of microtubules, typically arranged in parallel arrays, with predominantly subepithelial and subendothelial localization. By contrast, only some of the deposits in cryoglobulinaemic glomerulonephritis are organized, and they usually appear as short, curved or straight microtubules (FIG. $3 \mathrm{~d}$ ) with predominantly intraluminal and subendothelial localization ${ }^{52-54}$. Of note, organized deposits are not always observed in cryoglobulinaemic glomerulonephritis ${ }^{55}$.

The crystalline and/or inclusions category consists of LCPT, crystal-storing histiocytosis (CSH) and (cryo) crystalglobulinaemic glomerulonephritis ${ }^{56,57}$. LCPT occurs as crystalline and non-crystalline variants. In the crystalline variant, numerous light-chain crystals of various shapes are seen within proximal tubular cells, inside lysosomes or freely in the cytoplasm (FIGS 1, 3e). This variant is associated with $\kappa$ light-chain deposition and complete or partial Fanconi syndrome ${ }^{56-59}$. In the non-crystalline variant, proximal tubular cells are 


\section{CONSENSUS STATEMENT}
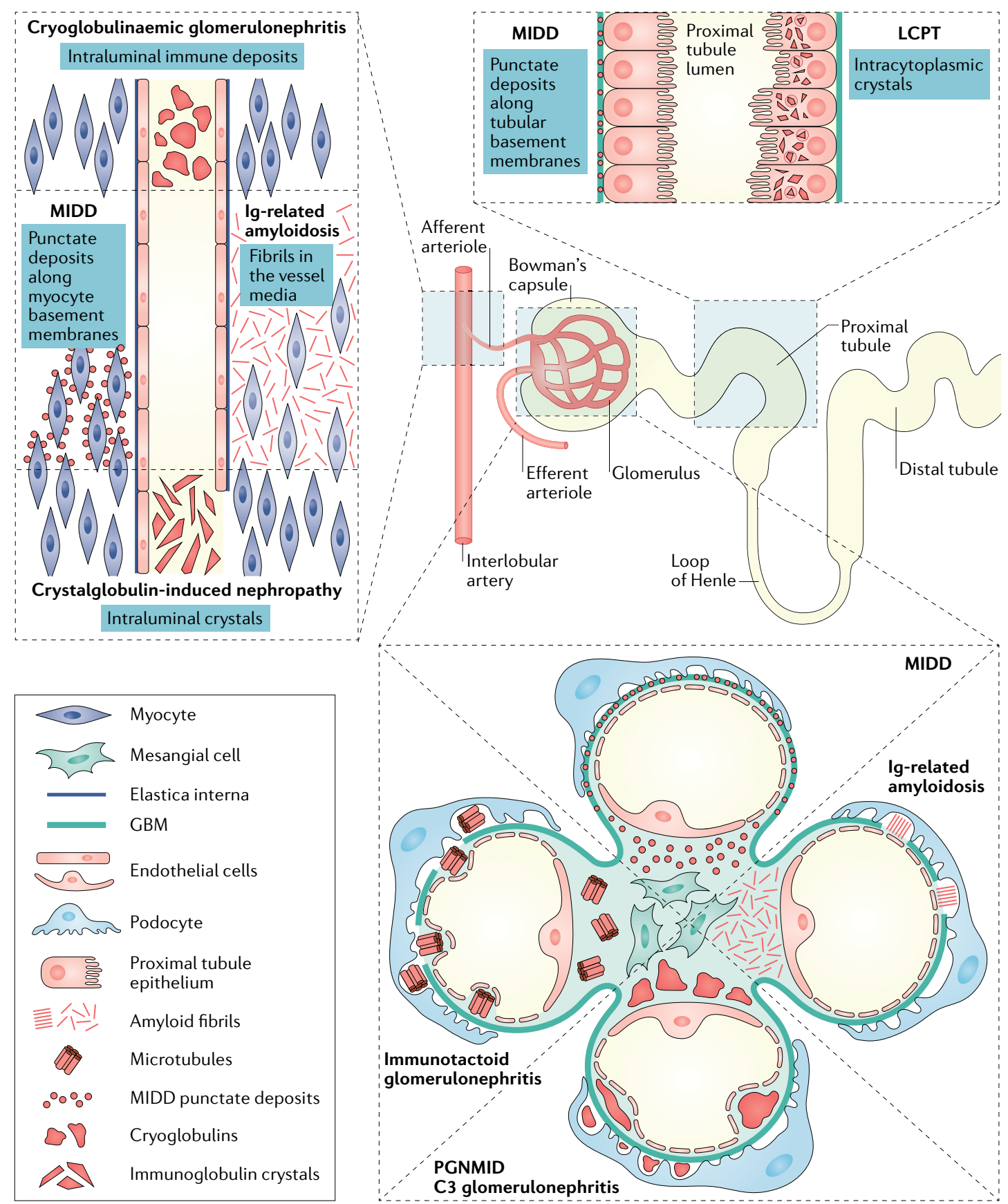

C3 glomerulonephritis

Fig. 1 | Localization of MGRS-associated renal lesions. Monoclonal gammopathy of renal significance (MGRS)-associated lesions can involve one or more renal compartments. In immunotactoid glomerulonephritis, C3 glomerulopathy and proliferative glomerulonephritis with monoclonal immunoglobulin deposits (PGNMID), MGRS-associated lesions involve only the glomeruli, whereas in light-chain proximal tubulopathy (LCPT), MGRS-associated lesions involve only the proximal tubules. MGRS-associated lesions in cryoglobulinaemic glomerulonephritis mainly involve the glomeruli but can occasionally affect blood vessels in the form of intravascular cryoglobulin thrombi or endovasculitis. Immunoglobulinrelated amyloidosis and monoclonal immunoglobulin deposition disease (MIDD) usually affect all renal compartments, including glomeruli, vessels and the tubulointerstitium. GBM, glomerular basement membrane.

distended and injured by the accumulation of numerous non-crystalline light-chain inclusions within lysosomes. This variant is typically associated with $\lambda$ rather than $\kappa$ light-chain deposition, and Fanconi syndrome is uncommon $^{56-59}$. Rarely, non-crystalline LCPT can mimic acute tubular necrosis or acute interstitial nephritis ${ }^{57,59}$. In patients with $\mathrm{CSH}$, light-chain crystals are often seen in renal histiocytes as well as in proximal tubular cells ${ }^{60}$ (FIG. 3f) and can have a widespread extrarenal distribution, including in bone marrow, lymph nodes, lungs, thyroid, parotid gland, cornea, synovium, skin, subcutaneous fat, stomach, liver and brain ${ }^{61-64}$. Finally, (cryo)crystalglobulinaemic glomerulonephritis is a rare monoclonal gammopathy characterized by immunoglobulin thrombi in 


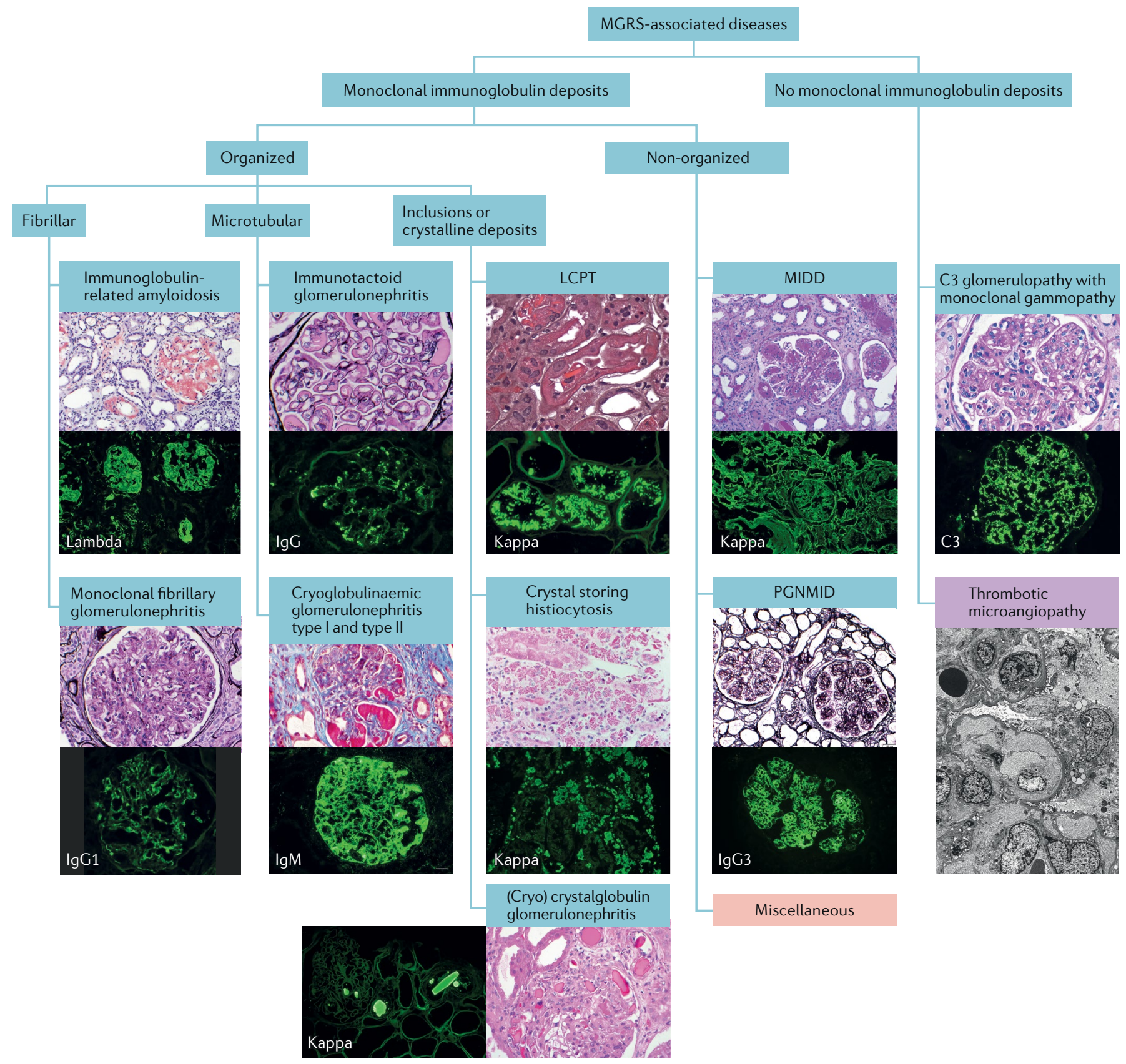

Fig. 2 | Categorization of MGRS-associated renal lesions. Monoclonal gammopathy of renal significance (MGRS)associated renal lesions (blue boxes) are initially separated by the presence or absence of monoclonal immunoglobulin deposits in kidney biopsy samples. They are further subcategorized by the ultrastructural characteristics of the deposits into organized and non-organized. Organized deposits are further subdivided into fibrillar, microtubular and inclusions or crystalline categories. Images of typical histological sections stained with haematoxylin and eosin (HEE), periodic acidSchiff or Masson trichrome stain and Congo red (top) are paired with immunofluorescence studies of frozen tissue sections (bottom) to reveal the specific immunoglobulin species. Pink box: the miscellaneous category represents polyclonal glomerulopathies that sometimes present with monoclonal immunoglobulin deposits, such as monotypic membranous nephropathy and monotypic anti-glomerular basement membrane disease. Purple box: thrombotic microangiopathy currently has a provisional status as an MGRS-associated lesion pending further evidence. Because this lesion has no immunoglobulin deposits and is best identified by electron microscopy, the immunofluorescence and H\&E stained sections were replaced by an electron micrograph. LCPT, light-chain proximal tubulopathy; MIDD, monoclonal immunoglobulin deposition disease; PGNMID, proliferative glomerulonephritis and monoclonal immunoglobulin deposits.

the arterioles and glomerular capillaries. These thrombi exhibit a crystalline structure or periodicity on electron microscopy ${ }^{65}$. In some patients, the crystallization process in the periphery is precipitated by cold exposure, termed cryocrystalglobulinaemia ${ }^{66}$. Mesangial and endocapillary hypercellularity is often absent ${ }^{65,66}$. As in cryoglobulinaemia, intravascular crystal deposition results in small-vessel occlusion, thrombosis and/or inflammatory 

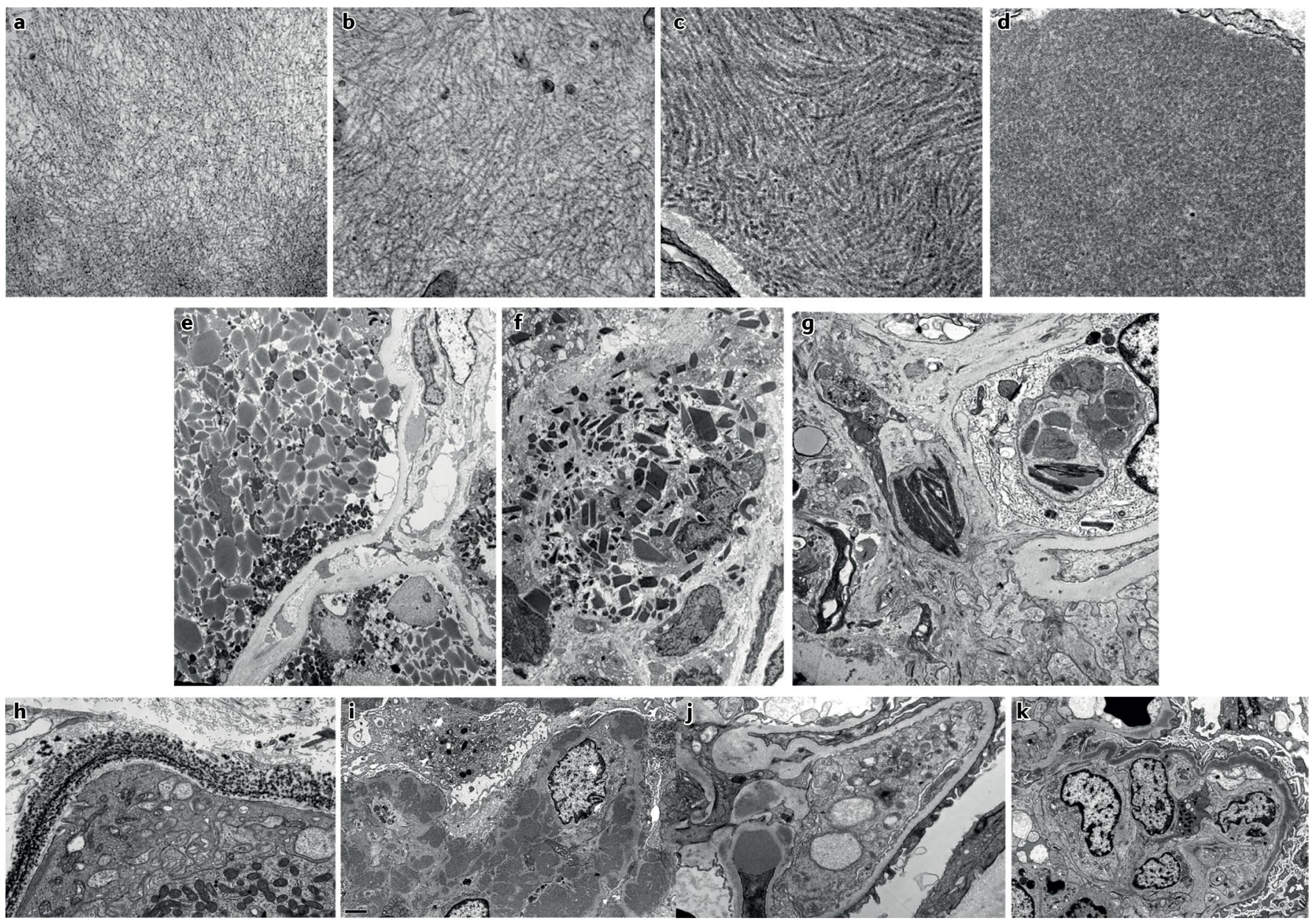

Fig. 3 | Ultrastructural appearance of MGRS-associated lesions. Top row: electron microscopy images showing fibrillar or microtubular deposits. a | Small randomly oriented fibrils of mean thickness $10 \mathrm{~nm}$ in a patient with immunoglobulin light-chain- $\kappa$ amyloidosis (original magnification $\times 49,000$ ). $\mathbf{b} \mid$ Randomly oriented fibrils with mean thickness of $15 \mathrm{~nm}$ in a patient with fibrillary glomerulonephritis (original magnification $\times 52,000$ ). $\mathbf{c} \mid$ Deposits composed of microtubules with hollow centres organized in parallel arrays and with a mean thickness of $26 \mathrm{~nm}$ in a patient with immunotactoid glomerulopathy (original magnification $\times 49,500$ ). $\mathbf{d} \mid$ Focal deposits composed of short microtubules with hollow centres with a mean thickness of $29 \mathrm{~nm}$ in a patient with cryoglobulinaemic glomerulonephritis (original magnification $\times 40,000$ ). Centre row: electron microscopy images showing crystals or inclusions. e | Proximal tubular cells filled with moderately electron-dense, light-chain crystals that have rod and rhomboid shapes in a patient with crystalline light-chain proximal tubulopathy. The crystals are predominantly free within the cytoplasm, not membrane bound (original magnification $\times 2,700)$. $\mathbf{f} \mid$ Numerous light-chain crystals with rod, rectangle or rhomboid shapes within the cytoplasm of interstitial infiltrating histiocytes in a patient with crystal-storing histiocytosis (original magnification $\times 4,200$ ). $\mathbf{g} \mid$ Needle-shaped, electron-dense crystals in the mesangium and within phagolysosomes of infiltrating inflammatory cells in a patient with cryocrystalglobulinaemia (original magnification $\times 9,300$ ). The crystals showed monotypic staining for $\lg \mathrm{G}$ and $\kappa$ light chains on pronase immunofluorescence. Bottom row: electron microscopy images showing non-organized deposits. $\mathbf{h} \mid$ Finely granular, highly electron-dense deposits along a tubular basement membrane in a patient with light-chain deposition disease (original magnification $\times 15,000$ ). i | Large, discrete (mesangial, subendothelial and subepithelial) granular, electrondense deposits in a patient with proliferative glomerulonephritis with monoclonal immunoglobulin deposits (original magnification $\times 6,000$ ). $\mathbf{j} \mid$ Mesangial deposits and a hump-shaped subepithelial deposit located overlying the glomerular basement membrane reflection over the mesangium in a patient with $\mathrm{C} 3$ glomerulonephritis associated with monoclonal gammopathy (original magnification $\times 9,300$ ). $\mathbf{k}$ |'Sausage-like' thickening of the glomerular basement membrane associated with highly electron-dense intramembranous deposits in a patient with dense deposit disease associated with monoclonal gammopathy (original magnification $\times 4,800$ ). MGRS, monoclonal gammopathy of renal significance.

vasculitis $^{65,67}$. Renal biopsy samples in patients with (cryo) crystalglobulinaemia reveal large extracellular crystals within glomerular capillaries and arterioles, which are frequently associated with fibrin thrombi and inflammation. Intracellular crystals can also be seen in patients with cryocrystalglobulinaemia (FIG. 3g).
Lesions with non-organized deposits. Non-organized monoclonal immunoglobulin deposits are seen in patients with MIDD and those with proliferative glomerulonephritis with monoclonal immunoglobulin deposits (PGNMID). MIDD comprises a group of diseases characterized by deposition of light chains, heavy 
chains or both light and heavy chains ${ }^{68-70}$. In MIDD (FIGS 1, 3h), linear punctate deposits are seen along both the glomerular basement membrane (GBM) and the tubular basement membrane (and occasionally extrarenally). By contrast, in PGNMID, deposits are confined to the glomeruli, where they are present in the mesangium and subendothelial space and occasionally in the subepithelial space (FICS 1, 3i). In addition, the deposits seen in PGNMID contain only intact immunoglobulins ${ }^{24,71}$, whereas those seen in heavy-chain MIDD or light-and-heavy-chain MIDD typically lack the first constant domain of the immunoglobulin ${ }^{24,70,71}$. In most patients, PGNMID is IgG3-driven, whereas truncated IgG1 is the most frequent immunoglobulin deposited in heavy-chain $\mathrm{MIDD}^{70,72}$. However, PGNMID can also be IgA-driven or (rarely) IgM-driven ${ }^{72,73}$.

Lesions without deposits. Not all MGRS-associated renal lesions include monoclonal immunoglobulin deposits. The best example of an MGRS-associated disorder lacking such deposits is C3 glomerulopathy with monoclonal gammopathy, which includes both $\mathrm{C} 3$ glomerulonephritis and the rare entity of dense deposit disease. By definition, substantial renal immunoglobulin deposits will be absent in patients with $\mathrm{C} 3$ glomerulopathy, although $60-80 \%$ of patients aged $>50$ years with C3 glomerulopathy have a monoclonal gammopathy at the time of diagnosis ${ }^{74-76}$. This proportion far exceeds the expected rate in the general population ${ }^{75-77}$. Thus, although renal disease related to the monoclonal immunoglobulin can be demonstrated in only about $30 \%$ of patients affected by $\mathrm{C} 3$ glomerulopathy (in whom the monoclonal immunoglobulin acts as a C3 nephritic factor or anti-factor-H antibody), it should still be considered an MGRS-associated disorder ${ }^{25,75}$.

C3 glomerulonephritis and dense deposit disease are distinguished by their ultrastructural appearance: ill-defined, moderately electron-dense mesangial, subepithelial and subendothelial deposits are seen in C3 glomerulonephritis (FIG. 3j), whereas highly electron-dense 'sausage-like' intramembranous deposits and mesangial rounded nodular deposits are seen in dense deposit disease (FIG. 3k). Large 'hump-shaped' subepithelial deposits might be seen in either lesion ${ }^{25}$ (FIG. 3j). C3 glomerulonephritis is the most common form of $\mathrm{C} 3$ glomerulopathy with monoclonal gammopathy. Importantly, roughly $5-10 \%$ of patients with monoclonal gammopathy and findings on standard immunofluorescence (that is, conducted on frozen tissue) consistent with C3 glomerulonephritis will actually have a membranoproliferative glomerulonephritis with masked monoclonal deposits. These patients require additional immunofluorescence studies to be performed on protease-digested, paraffinembedded tissue for identification of the monoclonal immunoglobulin in the deposits ${ }^{78,79}$.

Lesions with provisional status. Thrombotic microangiopathy is the endothelial injury seen most commonly in microangiopathy with haemolytic anaemia (MAHA). Thrombotic microangiopathy and MAHA can occur concurrently in patients with monoclonal gammopathies, including $\mathrm{MM}$ and $\mathrm{WM}^{13,42,80,81}$.
The pathophysiology of these disorders is not entirely understood but might be related to the monoclonal immunoglobulin acting as an autoantibody against a complement regulatory protein ${ }^{82}$. The other lesion in this category is glomerular microangiopathy associated with polyneuropathy, organomegaly, endocrinopathy, monoclonal gammopathy and skin changes (POEMS) syndrome ${ }^{83,84}$. The glomerular microangiopathy seen in POEMS syndrome is associated with a monoclonal gammopathy, which is nearly always $\lambda$ light-chain type. However, the $\lambda$ light chain itself is usually absent from kidney biopsy samples. Instead, the lesion is a subacute to chronic glomerular thrombotic microangiopathy characterized by mesangial and endothelial cell proliferation, mesangiolysis, widening of the subendothelial zone and double contouring ${ }^{85}$. Interestingly, these patients show no evidence of MAHA. The renal lesions in POEMS syndrome are thought to be secondary to a cytokine-mediated endothelial cell injury, similar to that seen in myeloproliferative neoplasm-related glomerulopathy ${ }^{86}$.

Lesions classed as miscellaneous. The 'miscellaneous' subcategory of MGRS-associated lesions includes kidney diseases that are typically not associated with MGRS, such as anti-GBM disease secondary to a monoclonal gammopathy. The anti-GBM monoclonal antibody can be IgG or IgA ${ }^{87-89}$. In most patients with this disease, the anti-GBM antibody is not detectable in serum by commercially available enzyme-linked immunosorbent assay (ELISA) or multiplex flow immunoassays, which are designed to detect antibodies against only a $3 \mathrm{NC1}$. These patients experience frequent relapses and the disease recurs after kidney transplantation, which is not typical in patients with non-MGRS-associated anti-GBM disease ${ }^{87-89}$. A pattern of membranous nephropathy that is visually indistinguishable from that associated with polyclonal immunoglobulin-mediated membranous lesions on light microscopy and electron microscopy has been described in patients with monoclonal IgG deposits $^{90,91}$. Although the phospholipase A2 receptor (PLA2R) was identified as the target of the monoclonal IgG in a single patient included in a small study, a larger study found that only $26 \%$ of patients showed evidence of antibodies to PLA2R and that none of those patients had a lymphoproliferative disorder ${ }^{90,91}$. Finally, HenochSchönlein purpura with IgA nephropathy has very occasionally been reported in patients with IgA monoclonal gammopathy or $\mathrm{MM}^{92,93}$.

\section{Evaluation of suspected MGRS}

Owing to differences in clinical characteristics and therapy, it is essential to distinguish MGRS-associated disorders from kidney diseases that are unrelated to monoclonal immunoglobins $s^{10,12,24,28,94,95}$. In patients suspected of having MGRS, the evaluation starts with a kidney biopsy. If analysis of the biopsy sample identifies an MGRS-associated lesion, a haematological evaluation (including monoclonal immunoglobulin studies, clonal determination and cytogenetic analysis) should be performed. These steps are discussed in greater detail below. 
When to perform a renal biopsy. As MGRS is a haematological condition defined by its renal manifestations, a kidney biopsy is essential for its diagnosis. However, not every patient with a monoclonal gammopathy and kidney disease has MGRS. The frequency of MGUS is 3\% in people aged $>50$ years, $5 \%$ in persons aged $>70$ years and as high as $8 \%$ in men aged $>80$ years ${ }^{77}$. The prevalence of MGUS is two to three times higher in African Americans than in white individuals of the same population $^{96}$. The incidence of chronic kidney disease (CKD) also increases after age 60 years ${ }^{97}$. Therefore, the same patient could have both MGUS and CKD that are unrelated to each other. Studies from the same county in the United States found that the annual incidence of glomerular disease was approximately 1 per 100,000 individuals in the general population and that the prevalence of MGUS in people aged $>50$ years was $3.2 \%{ }^{77,98}$. A renal biopsy study of patients with clinically suspected MGUS found that $45 \%$ of these patients did not have an MGRS-associated kidney disorder; however, as additional disease entities related to monoclonal gammopathies have been identified after the publication of this article, the true value might be lower ${ }^{10}$.

Clinicians must balance the risks associated with underdiagnosis of potentially treatable conditions against those of complications from the biopsy procedure. However, patients with MGRS-associated renal lesions (including amyloidosis) do not experience any increase in the risk of bleeding after kidney biopsy (which remains about $4 \%)^{99,100}$. Thus, performing a kidney biopsy in a patient with diabetes and rapidly progressive loss of renal function or increasing proteinuria is reasonable, especially if their diabetes is well controlled and/or evidence of extrarenal microvascular disease is absent. Because MGUS is uncommon in individuals aged $<50$ years (and is especially rare in those aged $<40$ years), its presence in people aged $<50$ years, when accompanied by renal manifestations, deserves a thorough evaluation. Older age ( $\geq 70$ years) should not discourage biopsy as most MGRS-related renal diseases occur in patients aged $>50$ years. In young and physically fit patients who are eligible for kidney transplantation, a kidney biopsy should be performed provided the kidneys are not markedly shrunken. Transjugular kidney biopsy is an option in high-risk patients from whom it would otherwise be difficult to obtain kidney tissue $\mathrm{e}^{101,102}$.

Renal biopsy evaluation. The diagnosis of MGRS associated lesions requires the integration of morphological alterations seen on light microscopy with the findings of immunohistochemistry (immunofluorescence or immunoperoxidase) and transmission electron microscopy studies, as well as correlation with the patient's medical history and laboratory findings. In some patients, ancillary techniques are needed to establish the diagnosis, including protease immunofluorescence, ultrastructural immunogold labelling and laser microdissection followed by liquid chromatography and mass spectrometry (LC-MS). A detailed description of our consensus recommendations for renal biopsy and the indications for ancillary techniques is provided in TABLE 2. Our recommended approach to renal biopsy analysis in patients suspected to have MGRS is provided in FIG. 4.

To confirm the monotypic nature of the immunoglobulin deposits, immunofluorescence staining for IgG subclasses should be performed in biopsy samples from patients with glomerular disorders related to deposition of an intact monoclonal IgG (such as PGNMID, immunotactoid glomerulopathy, type I cryoglobulinaemic glomerulonephritis and monoclonal membranous nephropathy) or of a truncated monoclonal heavy chain (such as heavy-chain deposition disease, heavy-and-light-chain deposition disease or heavy-chain amyloidosis). Of note, IgG subclass restriction is not sufficient by itself to establish monoclonality, as some non-MGRS glomerular diseases - such as PLA2R-associated polyclonal membranous nephropathy and non-monoclonal fibrillary glomerulonephritis commonly show staining restricted to one IgG subclass but positive staining for both $\kappa$ and $\lambda$ light chains ${ }^{23,103}$. Identification of complement $\mathrm{C} 1 \mathrm{q}$ and/or $\mathrm{C} 3$ proteins within the monotypic renal deposits might reveal the cause of hypocomplementaemia in patients with MGRSassociated lesions such as PGNMID, immunotactoid glomerulonephritis, type I cryoglobulinaemic glomerulonephritis, C3 glomerulonephritis and heavy-chain or heavy-and-light-chain deposition disease $\mathrm{e}^{23,24,51,52,68,103,104}$.

Electron microscopy is often necessary to identify the specific MGRS-associated lesion. Ideally, electron microscopy should be performed on $2-3 \%$ glutaraldehyde-fixed tissue. If glutaraldehyde-fixed tissue is not available or lacks glomeruli, formalin-fixed, paraffin-embedded tissue samples can be reprocessed for electron microscopy. Although reprocessing is usually associated with various artefacts, the immune deposits generally remain sufficiently intact to permit an accurate assessment of their location and structure. Electron microscopy of frozen tissue samples or tissue fixed in Zenker or B5 fixatives is not recommended owing to its generally very poor ultrastructural preservation. In patients with monoclonal gammopathy, at least two glomeruli should be studied ultrastructurally as glomerular deposits can be sparse and only a portion of the deposits show substructural features. For example, in most patients with cryoglobulinaemic glomerulonephritis, only a small number of deposits (most commonly intraluminal ones) exhibit the microtubular substructure that is so useful in establishing the diagnosis ${ }^{53}$. In patients with monoclonal gammopathy, a directed search for intratubular cytoplasmic crystals or inclusions by electron microscopy is of paramount importance, as these features can be overlooked by light microscopy and standard immunofluorescence studies on frozen tissue. Additionally, in some patients with classic MIDD, the characteristic punctate, powdery, electron-dense deposits can be found only in small sections of the tubular basement membranes. Therefore, a thorough search for tubular basement membrane deposits by electron microscopy is necessary to distinguish classic MIDD from MIDD identified by immunofluorescence only ${ }^{69}$. Ultrastructural immunogold labelling is a sensitive technique that can assist in the histopathological diagnosis of MGRS-associated lesions, such as AL amyloidosis, 
Table 2 | Consensus recommendations for the evaluation of MGRS-associated disorders

$\begin{array}{ll}\text { Modality } & \text { Recommendations } \\ \text { Kidney biopsy } & \text { Recommended in the following patients: } \\ \text { - Those with monoclonal gammopathy and unexplained kidney disease } & \text { - Those with known risk factors for chronic kidney disease but an atypical clinical course } \\ \text { - Patients with kidney disease and monoclonal gammopathy aged <50 years }\end{array}$

Renal amyloid typing by liquid chromatography and mass spectrometry

Flow cytometry or other immunotyping

Immunohistochemistry

Mutational analysis

FISH
Recommended in the following situations:

- When frozen tissue for immunofluorescence is not available

- Negative immunofluorescence staining for $\kappa$ and $\lambda$ light chains, with negative immunoperoxidase staining for SAA and LECT2

- Equal staining for $\kappa$ and $\lambda$ light chains by immunofluorescence

- Bright staining for $\mathrm{lgG}$ and/or IgA by immunofluorescence

- Equivocal Congo red staining

- To enable distinction between AHL amyloidosis and congophilic fibrillary glomerulonephritis

- Neoplastic plasma cells frequently show aberrant loss of CD45 and CD19, as well as aberrant expression of CD56 and CD117; therefore, these markers (in addition to $\kappa$ and $\lambda$ light chains and CD38) are useful in identifying small plasma cell clones

- Including CD5 and CD20 in the immunophenotyping of B cells can frequently separate small clones from polytypic cells

- The most sensitive assay available at a given institution should be used. Although there is no established gold standard, many laboratories have the capability to determine minimal residual disease in MGRS at a sensitivity of $10^{-4}$ to $10^{-6}$ monoclonal cells. The sensitivity of flow cytometry immunophenotyping depends on the total number of collected cells, the number of antibodies used to find an aberrant phenotype, the phenotype of the abnormal clone and sample quality

- Immunohistochemistry of bone marrow biopsy samples has a low sensitivity for detecting $\kappa$-expressing and $\lambda$-expressing plasma cells and could be useful only if there is a major plasma cell clone and a lack of polyclonal plasma cells

- Immunohistochemistry might be useful in the evaluation of atypical lymphoid infiltrates, particularly if flow cytometry is not available or infiltrates are very focal

- If an abnormal clone is detected, the light-chain isotype should be compared with that present in renal lesions and additional information should be obtained

The MYD88 L265P mutation is found in over $90 \%$ of patients with lymphoplasmacytic lymphoma or Waldenström macroglobulinaemia but in only $40-60 \%$ of individuals with lgM MGUS

Cyclin D1 FISH with immunostaining for CD10, BCL2 and BCL6 to subclassify diffuse large cell lymphoma, and prognostic FISH panels for MM and CLL, can also be useful

AHL, immunoglobulin A heavy-and-light chain; CLL, chronic lymphocytic leukaemia; CSH, crystal-storing histiocytosis; FISH, fluorescence in situ hybridization; LCPT, light-chain proximal tubulopathy; LECT2, leukocyte cell-derived chemotaxin 2; MGRS, monoclonal gammopathy of renal significance; MGUS, monoclonal gammopathy of undetermined significance; MM, multiple myeloma; NA, not applicable; SAA, serum amyloid A protein.

MIDD, LCPT and CSH, by confirming the location and composition of monoclonal deposits, but it is not widely available ${ }^{35,60,70,105-107}$.

Laser microdissection followed by LC-MS is currently the gold standard for amyloid typing but is available in only a few specialized centres. In renal pathology laboratories that routinely perform immunofluorescence studies on native kidney biopsy samples, LC-MS is essential for typing renal amyloidosis in about $15 \%$ of patients ${ }^{43,108}$. LC-MS is crucial for the diagnosis of rare hereditary forms of renal amyloidosis that cannot be typed by immunofluorescence, but it is also important to distinguish $\mathrm{AH}$ and AHL amyloidoses from nonimmunoglobulin amyloidoses associated with nonspecifically entrapped immunoglobulins (particularly AA amyloidosis) and from fibrillary glomerulonephritis ${ }^{49,109}$.
LC-MS can also be useful in the diagnosis of MGRSassociated lesions other than immunoglobulin amyloidosis when immunofluorescence studies are not available or have negative findings. An example of the latter situation is $\mathrm{IgD}$ heavy-chain deposition disease, which is generally missed by immunofluorescence studies because an IgD antibody is not included in the routine immunofluorescence panel ${ }^{110}$.

Monoclonal immunoglobulin testing. Once the diagnosis of an MGRS-associated lesion has been established, a search for the culprit monoclonal immunoglobulin should be undertaken (if it has not been identified already). Protein electrophoresis analyses of serum and urine samples are the first tests performed ${ }^{111}$. Although its sensitivity is inferior to that of some other tests discussed 


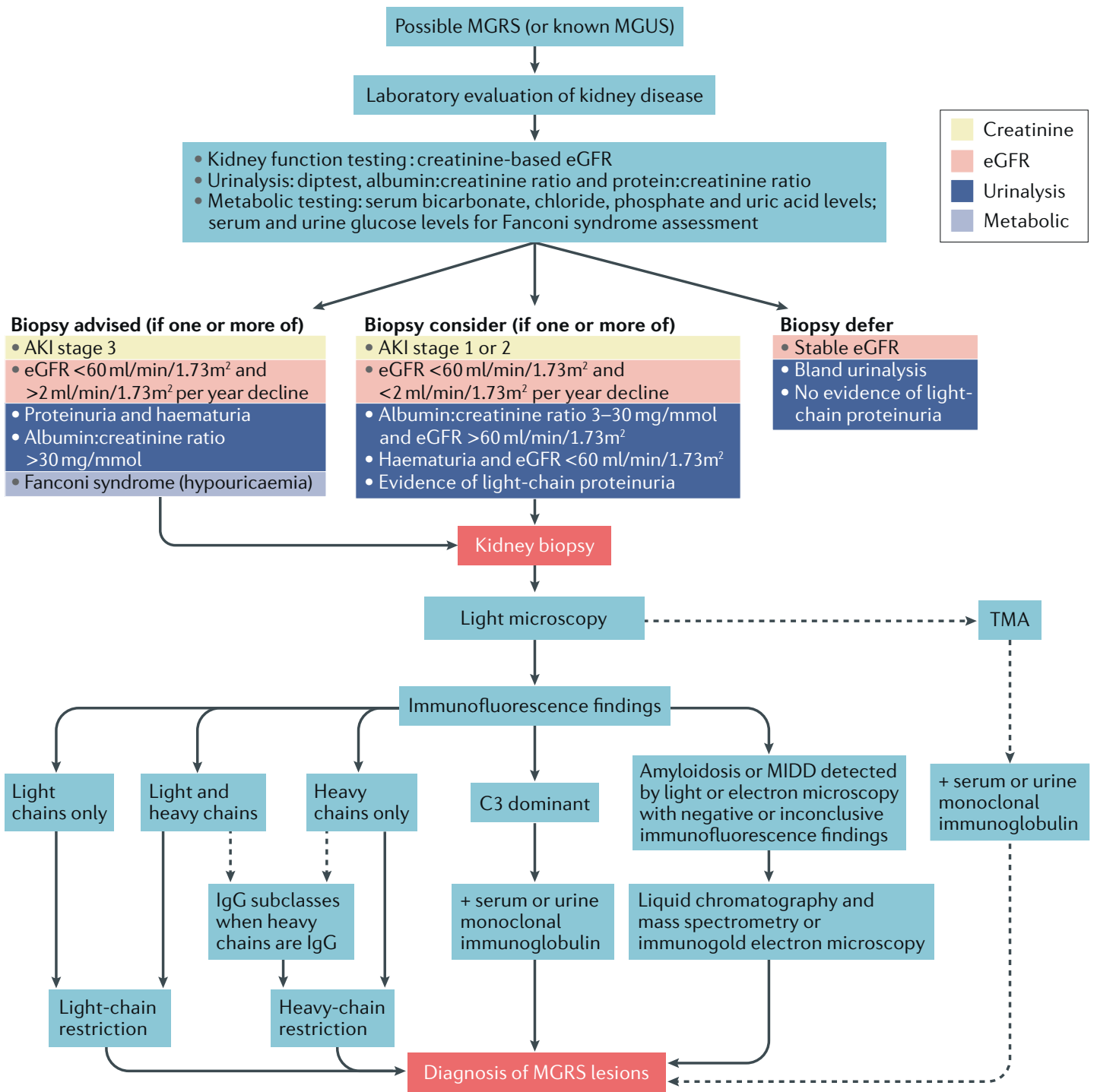

Fig. 4 | Algorithm for renal biopsy evaluation in patients suspected to have MGRS. Kidney biopsy analysis in patients suspected to have monoclonal gammopathy of renal significance (MGRS) should include light microscopy (including staining the paraffin sections with haematoxylin and eosin, periodic acid-Schiff, Masson trichrome, Jones methenamine silver and Congo red). Immunofluorescence studies conducted on frozen tissue should include staining for $\lg \mathrm{G}, \lg \mathrm{M}, \lg \mathrm{A}$, $\mathrm{C} 1 q, \mathrm{C} 3$ and $\kappa$ and $\lambda$ light chains. Finally, transmission election microscopy should be conducted. This standard renal biopsy approach enables diagnosis of MGRS in the majority of affected patients. In some individuals, ancillary techniques are needed to establish the diagnosis, including mass spectrometry, immunogold electron microscopy, immunofluorescence staining for $\lg \mathrm{G}$ subtypes and paraffin immunofluorescence. The indications for these ancillary techniques are detailed in TABLE 2. AKI, acute kidney injury; eGFR, estimated glomerular filtration rate; MGUS, monoclonal gammopathy of undetermined significance; MIDD, monoclonal immunoglobulin deposition disease; TMA, thrombotic microangiopathy.

here, serum protein electrophoresis is quantitative, easy to perform and inexpensive. Urine protein electrophoresis is less sensitive than serum protein electrophoresis but provides the total protein level, urinary albumin level and globular protein (monoclonal immunoglobulin or light chain) component - parameters that are necessary for diagnosis, prognostication and response assessment ${ }^{112-114}$. Immunofixation of a serum sample and of a concentrated urine aliquot from a $24 \mathrm{~h}$ collection should also be done because this test is more sensitive than protein electrophoresis. Immunofixation is necessary for the identification and typing of monoclonal immunoglobulins, as well as for the determination of a complete response $\mathrm{e}^{113,115}$.
Immunoblotting is a highly sensitive technique that can detect small amounts of monoclonal immunoglobulin, characterize the distribution of IgG heavy-chain subclasses and detect deletion of the first constant domain, the hallmark of heavy-chain deposition disease and $\mathrm{AH}$ amyloidosis ${ }^{70}$. However, this technique is not widely available.

Another critical test is the serum free light-chain assay, which detects unbound free light chains ${ }^{113}$. This assay measures $\kappa$ and $\lambda$ free light chains independently and can be used to determine the $\kappa: \lambda$ free light-chain ratio. Clonality can be inferred from an abnormal $\kappa: \lambda$ free light-chain ratio: a high ratio indicates a $\kappa$ clone whereas a low ratio indicates a $\lambda$ clone. Because free light 
chains are cleared by the kidney, impaired renal function alters the free light-chain concentration. The 'normal' free light-chain ratio, $0.26-1.65$, can rise to $0.34-3.10$ in patients with severe renal impairment (CKD stage 5 or greater), but small declines in renal function can also impair free light-chain clearance ${ }^{116}$. Knowing which serum free light-chain assay is being used by the laboratory is extremely important, as at least two major assays are currently on the market. Not only are the results of these assays mathematically inconvertible, but the effects of renal impairment differ between these assays; the evidence suggests that the $\mathrm{N}$ Latex assay is less affected than the FreeLite assay by impaired renal function ${ }^{117}$. Thus, the same assay must be used to monitor a particular patient throughout their treatment. Moreover, given that the two assays have different performance characteristics, free light-chain levels might need to be checked using the other assay if the first result is negative. In addition, serum immunofixation might be more helpful than serum free light-chain assays in diseases associated with an intact monoclonal immunoglobulin (such as PGNMID $)^{72}$. Finally, although antibodies for use in urinary light-chain assays have been developed, these assays have not been validated and should not be used to quantify the amount of light chain (Bence Jones protein) in a $24 \mathrm{~h}$ urine specimen (which should instead be measured by urine protein electrophoresis, as previously stated $)^{118}$.

Identification of the culprit monoclonal immunoglobulin has important diagnostic and prognostic consequences. The monoclonal immunoglobulin detected in serum and/or urine must match that found in immunoglobulin deposits in the kidney ${ }^{35}$; if the immunoglobulin found in renal deposits differs from that found in the circulation, the monoclonality of the putative culprit immunoglobulin is called into question. Although the serum M-spike concentration and serum free light-chain assay results have both diagnostic and prognostic importance, the correlation between the results of these tests and the severity or type of kidney disease is less well established.

\section{Clonal identification}

The diagnosis of MGRS should generally be established before obtaining a haematological consultation. The focus of the haematologist and/or oncologist and haematopathologists should be clonal identification, which is central to the management of patients with MGRS. The only exception is when the patient has already been diagnosed as having MM, WM or CLL, which eliminates the need for a kidney biopsy (because treatment will be initiated regardless of the kidney lesions present). Clonal identification is essential because the same kidney diseases can occur in different haematological disorders (TABLE 3). Of note, although a pathological clone can be identified in virtually every patient with AL amyloidosis or MIDD, such clones are often difficult to detect in other diseases. For example, the chance of identifying the pathological clone falls below $17 \%$ for patients who do not have a detectable monoclonal immunoglobulin on immunofixation studies ${ }^{72}$, and only $20-30 \%$ of patients with PGNMID have a detectable circulating monoclonal immunoglobulin ${ }^{24,72}$. As treatment differs according

Table 3 | Renal lesions associated with monoclonal gammopathy

\begin{tabular}{|c|c|c|c|c|c|c|}
\hline \multirow[t]{2}{*}{ Lesion } & \multicolumn{6}{|c|}{ Proportion of lesions (\%) } \\
\hline & $\begin{array}{l}\text { Monoclonal } \\
\text { immuno- } \\
\text { globulin } \\
\text { deposits }\end{array}$ & $\begin{array}{l}\text { Detectable } \\
\text { monoclonal } \\
\text { immuno- } \\
\text { globulin }\end{array}$ & MM & MGRS & Other ${ }^{a}$ & Refs \\
\hline Light-chain cast nephropathy & 100 & 100 & 99 & 0 & $\sim 1$ & 2,4,11,13 \\
\hline Immunoglobulin-related amyloid amyloidosis & 96 & 99 & 16 & 80 & $1-4$ & $43,113,128,129$ \\
\hline MIDD & 100 & 100 & $0-20$ & $78-100$ & $1-2$ & $29,31,68,130,131$ \\
\hline Light-chain proximal tubulopathy & 100 & $97^{\mathrm{b}}$ & $12-33$ & $61-80$ & $3-8$ & 32,56,58,132 \\
\hline Cryoglobulinaemic (type I) glomerulonephritis & 100 & $90-100$ & $6-8$ & $47-52$ & $24-56$ & $133-136$ \\
\hline Cryoglobulinaemic (type II) glomerulonephritis & 100 & 49 & 0 & 20 & 7 & 133-136 \\
\hline PGNMID & 100 & $30-32$ & 4 & 96 & $\sim 1$ & 24,72 \\
\hline Crystal-storing histiocytosis & 83 & 90 & 33 & 8 & 50 & 137 \\
\hline $\begin{array}{l}\text { Cryocrystalglobulin or crystalglobulin } \\
\text { nephropathy }\end{array}$ & 91 & 82 & 61 & 18 & 4 & 138 \\
\hline Immunotactoid glomerulonephritis & $69-93$ & $63-71$ & $0-13$ & $25-50$ & $25-50$ & 23,51 \\
\hline $\begin{array}{l}\text { C3 glomerulopathy with monoclonal } \\
\text { gammopathyc }\end{array}$ & 0 & $28-83^{d}$ & $0-40^{d}$ & $40-90$ & $6-10$ & $25,74,75,104$ \\
\hline Monoclonal fibrillary glomerulonephritis & 100 & $7-17$ & $0-54$ & $55-98$ & $2-10$ & $44,47,139$ \\
\hline \multicolumn{7}{|c|}{ 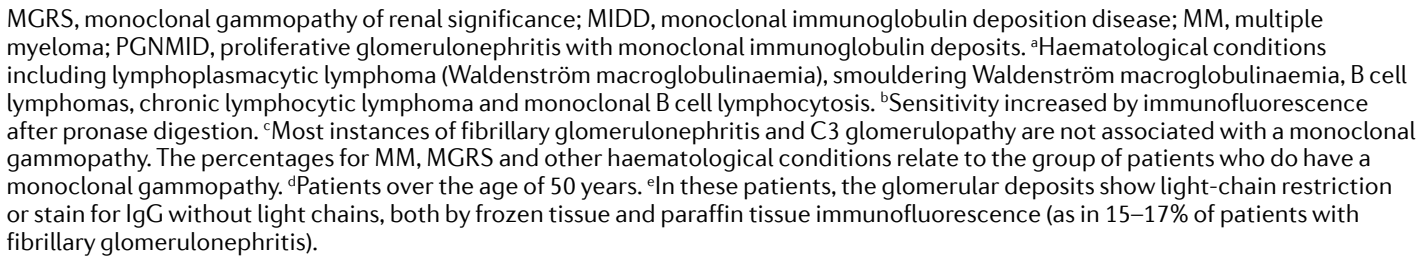 } \\
\hline
\end{tabular}


to whether the clone has a plasmacytic or lymphocytic nature, choosing the right agent is challenging if a clone cannot be identified.

Bone marrow aspiration and biopsy should be performed to evaluate MGRS in most patients, although in patients with CLL clones, the diagnosis could be made with peripheral blood flow cytometry. Morphological assessment should include quantification of the percentage of plasma cells (in plasma cell clones) and evaluation for the presence of atypical lymphoid or lymphoplasmacytic aggregates (in lymphoma clones) as well as amyloid deposits. In addition, ancillary studies - in particular, flow cytometry immunophenotyping, detection of minimal residual disease and cytogenetic and genetic evaluation of the clones - are helpful for the identification of small clones as well as for deriving treatment recommendations ${ }^{119-121}$. The myeloma fluorescent in situ hybridization (FISH) panel has shown increasing importance in guiding the treatment of patients with plasma cell dyscrasias. For example, patients with AL amyloidosis featuring translocation $\mathrm{t}(11 ; 14)$ have inferior responses to bortezomib-based therapy, whereas those with gain of chromosome 1q21 show poorer responses to melphalan plus dexamethasone ${ }^{99,100,122-124}$ (versus patients without these genetic variants). These findings highlight the importance of performing the myeloma FISH panel on all bone marrow biopsy samples from patients with plasma cell dyscrasia.

If bone marrow evaluation does not reveal a clonal haematological disorder, the next step could be to perform imaging studies (such as CT with or without PET, or whole-body MRI) to look for a localized plasmacytoma or for lymphadenopathy in low-stage, low-grade lymphoma ${ }^{34,35}$. For patients suspected to have MM, whole-body CT with or without PET (or MRI) should be performed to look for bone disease ${ }^{125,126}$. Any suspicious lesions should be biopsied and enough material should be obtained to enable diagnostic and prognostic studies. Next-generation flow cytometry has been used in the measurement of minimal residual disease ${ }^{127}$. This technique might be helpful in patients suspected of having MGRS who have negative findings on traditional cytology or flow cytometry studies of bone marrow samples.

\section{Summary}

MGRS is a new classification of pathogenic clonal proliferative disorders that produce a nephrotoxic protein. The term MGRS was needed to improve the classification of these diseases for research purposes, and to accurately categorize them as pathological, so that government agencies could allocate the resources necessary for their treatment. The diagnosis of MGRS can be established only by performing a kidney biopsy that either demonstrates the presence of monotypic immunoglobulin deposits or infers their involvement in the case of C3 glomerulonephritis or thrombotic microangiopathy with a circulating monoclonal immunoglobulin. Clinicians will need to balance the risk of missing a diagnosis against those of the complications of renal biopsy; therefore, the judicious use of renal biopsy is important. Detection of a monoclonal immunoglobulin, in addition to helping to establish the diagnosis of MGRS, has diagnostic and prognostic value and is also used to predict treatment responses. Haematological evaluation might require peripheral blood flow cytometry, bone marrow biopsy and imaging studies to assess localized disease.

Published online 3 December 2018
1. Kyle, R. A. Monoclonal gammopathy of undetermined significance. Natural history in 241 cases. Am. J. Med. 64, 814-826 (1978)

2. Rajkumar, S. V. et al. International Myeloma Working Group updated criteria for the diagnosis of multiple myeloma. Lancet Oncol. 15, e538-e548 (2014).

3. Dimopoulos, M. A. et al. Update on treatment recommendations from the Fourth International Workshop on Waldenstrom's macroglobulinemia. J. Clin. Oncol. 27, 120-126 (2009).

4. Strati, P. \& Shanafelt, T. D. Monoclonal B cell lymphocytosis and early-stage chronic lymphocytic leukemia: diagnosis, natural history, and risk stratification. Blood 126, 454-462 (2015).

5. Bence Jones, $\mathrm{H}$. On a new substance occurring in the urine of a patient with mollities ossium. Philos. Trans. R. Soc. B. Biol. Sci. 138, 55-62 (1848).

6. Steensma, D. P. \& Kyle, R. A. A history of the kidney in plasma cell disorders. Contrib. Nephrol. 153, 5-24 (2007).

7. Von Rustizky, J. Multiples myelom. Deutsch. Z. Chir. 3, 162-172 (1873)

8. Decastello, A. Beitrage zur Kenntnis der BencJonesschen Albuminurie [German]. Z. Kin. Med. 67, 319-343 (1909)

9. Koss, M. N., Pirani, C. L. \& Osserman, E. F. Experimental Bence Jones cast nephropathy. Lab. Invest. 34, 579-591 (1976).

10. Paueksakon, P., Revelo, M. P., Horn, R. G., Shappell, S. \& Fogo, A. B. Monoclonal gammopathy: significance and possible causality in renal disease. Am. J. Kidney Dis. 42, 87-95 (2003)

11. Higgins, L. et al. Kidney involvement of patients with Waldenström macroglobulinemia and other IgM-producing B cell lymphoproliferative disorders. Clin. J. Am. Soc. Nephrol. 13, 1037-1046 (2018).
12. Chauvet, $\mathrm{S}$. et al. Kidney diseases associated with monoclonal immunoglobulin M-secreting B cell lymphoproliferative disorders: a case series of 35 patients. Am. J. Kidney Dis. 66, 756-767 (2015).

13. Vos, J. M. et al. Renal disease related to Waldenström macroglobulinaemia: incidence, pathology and clinical outcomes. Br. J. Haematol. 175, 623-630 (2016).

14. Strati, P. et al. Renal complications in chronic lymphocytic leukemia and monoclonal B cell lymphocytosis: the Mayo Clinic experience. Haematologica 100, 1180-1188 (2015).

15. Pozzi, C. et al. Light chain deposition disease with renal involvement: clinical characteristics and prognostic factors. Am. J. Kidney Dis. 42, 1154-1163 (2003).

16. Kyle, R. A. \& Bayrd, E. D. Amyloidosis: review of 236 cases. Medicine (Baltimore) 54, 271-299 (1975)

17. Solomon, A., Weiss, D. T. \& Kattine, A. A. Nephrotoxic potential of Bence Jones proteins. N. Engl. J. Med. 324, 1845-1851 (1991)

18. Sirac, C. et al. Animal models of monoclonal immunoglobulin-related renal diseases. Nat. Rev. Nephrol. 14, 246-264 (2018)

19. Merlini, G. \& Stone, M. J. Dangerous small B cell clones. Blood 108, 2520-2530 (2006).

20. Glavey, S. V. \& Leung, N. Monoclonal gammopathy: the good, the bad and the ugly. Blood Rev. 30, 223-231 (2016)

21. Gertz, M. A. et al. Clinical outcome of immunoglobulin light chain amyloidosis affecting the kidney. Nephrol. Dial Transplant. 24, 3132-3137 (2009).

22. Zand, L. et al. Clinical and prognostic differences among patients with light chain deposition disease, myeloma cast nephropathy and both. Leuk. Lymphoma 56, 3357-3364 (2015)
23. Bridoux, F. et al. Fibrillary glomerulonephritis and immunotactoid (microtubular) glomerulopathy are associated with distinct immunologic features. Kidney Int. 62, 1764-1775 (2002).

24. Nasr, S. H. et al. Proliferative glomerulonephritis with monoclonal IgG deposits. J. Am. Soc. Nephrol. 20. 2055-2064 (2009).

25. Chauvet, S. et al. Treatment of B cell disorder improves renal outcome of patients with monoclonal gammopathy-associated $\mathrm{C} 3$ glomerulopathy. Blood 129, 1437-1447 (2017)

26. Czarnecki, P. G. et al. Long-term outcome of kidney transplantation in patients with fibrillary glomerulonephritis or monoclonal gammopathy with fibrillary deposits. Kidney Int. 75, 420-427 (2009).

27. Leung, N. et al. Long-term outcome of renal transplantation in light-chain deposition disease. Am. J. Kidney Dis. 43, 147-153 (2004).

28. Said, S, M. et al. Proliferative glomerulonephritis with monoclonal immunoglobulin $\mathrm{G}$ deposits is associated with high rate of early recurrence in the allograft. Kidney Int. 94, 159-169 (2011).

29. Cohen, C. et al. Bortezomib produces high hematological response rates with prolonged renal survival in monoclonal immunoglobulin deposition disease. Kidney Int. 88, 1135-1143 (2015).

30. Hassoun, $\mathrm{H}$. et al. High-dose melphalan and auto-SCT in patients with monoclonal Ig deposition disease. Bone Marrow Transplant. 42, 405-412 (2008).

31. Sayed, R. H. et al. Natural history and outcome of light chain deposition disease. Blood 126, 2805-2810 (2015).

32. Vignon, M. et al. Current anti-myeloma therapies in renal manifestations of monoclonal light chainassociated Fanconi syndrome: a retrospective series of 49 patients. Leukemia 31, 123-129 (2017). 
33. Leung, N. et al. Monoclonal gammopathy of renal significance: when MGUS is no longer undetermined or insignificant. Blood 120, 4292-4295 (2012).

34. Fermand, J. P. et al. How I treat monoclonal gammopathy of renal significance (MGRS). Blood 122 3583-3590 (2013)

35. Bridoux, F. et al. Diagnosis of monoclonal gammopathy of renal significance. Kidney Int. 87 698-711 (2015)

36. Kyle, R. A. et al. Progression in smoldering Waldenström macroglobulinemia: long-term results. Blood 119, 4462-4466 (2012).

37. Kyle, R. A. $\&$ Greipp, P. R. Smoldering multiple-myeloma. N. Engl. J. Med. 302, 1347-1349 (1980)

38. Li, S. J. et al. Renal involvement in non-Hodgkin lymphoma: proven by renal biopsy. PLOS ONE 9 , e95190 (2014).

39. Poitou-Verkinder, A. L. et al. The spectrum of kidney pathology in B cell chronic lymphocytic leukemia/ small lymphocytic lymphoma: a 25-year multicenter experience. PLOS ONE 10, e0119156 (2015).

40. Da'as, N. et al. Kidney involvement and renal manifestations in non-Hodgkin's lymphoma and lymphocytic leukemia: a retrospective study in 700 patients. Eur. J. Haematol. 67, 158-164 (2001).

41. Luciano, R. L. \& Brewster, U. C. Kidney involvement in leukemia and lymphoma. Adv. Chronic Kidney Dis. 21 27-35 (2014).

42. Ravindran, A., Go, R. S., Fervenza, F. C. \& Sethi, S. Thrombotic microangiopathy associated with monoclonal gammopathy. Kidney Int. 91, 691-698 (2017).

43. Said, S. M. et al. Renal amyloidosis: origin and clinicopathologic correlations of 474 recent cases. Clin. J. Am. Soc. Nephrol. 8, 1515-1523 (2013).

44. Nasr, S. H. et al. Fibrillary glomerulonephritis: a report of 66 cases from a single institution. Clin. J. Am. Soc. Nephrol. 6, 775-784 (2011)

45. Gibier, J. B. et al. Intratubular amyloid in light chain cast nephropathy is a risk factor for systemic light chain amyloidosis. Mod. Pathol. 31, 452-462 (2017).

46. Grove, P., Neale, P. H., Peck, M., Schiller, B. $\&$ Haas, M. Monoclonal immunoglobulin $\mathrm{G}_{1}-\mathrm{k}$ fibrillary glomerulonephritis. Mod. Pathol. 11, 103-109 (1998).

47. Rosenstock, J. L. et al. Fibrillary and immunotactoid glomerulonephritis: distinct entities with different clinical and pathologic features. Kidney Int. 63 1450-1461 (2003)

48. Nasr, S. H. et al. DNAJB9 is a specific immunohistochemical marker for fibrillary glomerulonephritis. Kidney Int. Rep. 3, 56-64 (2018).

49. Dasari, S. et al. DnaJ heat shock protein family $\mathrm{B}$ member 9 is a novel biomarker for fibrillary $\mathrm{GN}$ J. Am. Soc. Nephrol. 29, 51-56 (2018).

50. Alexander, M. P. et al. Congophilic fibrillary glomerulonephritis: a case series. Am. J. Kidney Dis 72, 325-336 (2018).

51. Nasr, S. H. et al. Immunotactoid glomerulopathy: clinicopathologic and proteomic study. Nephrol. Dial Transplant. 27, 4137-4146 (2012).

52. Karras, A. et al. Renal involvement in monoclonal (type I) cryoglobulinemia: two cases associated with $\lg \mathrm{G}_{3} \mathrm{~K}$ cryoglobulin. Am. J. Kidney Dis. 40, 1091-1096 (2002).

53. Ojemakinde, K., Turbat-Herrera, E. A., Zeng, X., Gu, X $\&$ Herrera, G. A. The many faces of cryoglobulinemic nephropathy: a clinico-pathologic study of 47 cases with emphasis on the value of electron microscopy. Ultrastruct. Pathol. 38, 367-376 (2014).

54. Heher, E. C., Rennke, H. G., Laubach, J. P. \& Richardson, P. G. Kidney disease and multiple myeloma. Clin. J. Am. Soc. Nephrol. 8, 2007-2017 (2013).

55. Nasr, S. H. et al. Dysproteinemia, proteinuria, and glomerulonephritis. Kidney Int. 69, 772-775 (2006).

56. Stokes, M. B. et al. Light chain proximal tubulopathy: clinical and pathologic characteristics in the modern treatment era. J. Am. Soc. Nephrol. 27, 1555-1565 (2015).

57. Kapur, U., Barton, K., Fresco, R., Leehey, D. J. $\&$ Picken, M. M. Expanding the pathologic spectrum of immunoglobulin light chain proximal tubulopathy Arch Pathol. Lab. Med. 131, 1368-1372 (2007).

58. Larsen, C. P. et al. The morphologic spectrum and clinical significance of light chain proximal tubulopathy with and without crystal formation. Mod. Pathol. 24, 1462-1469 (2011)

59. Herrera, G. A. Proximal tubulopathies associated with monoclonal light chains: the spectrum of clinicopathologic manifestations and molecular pathogenesis. Arch. Pathol. Lab. Med. 138 1365-1380 (2014).
60. El Hamel, C. et al. Crystal-storing histiocytosis with renal Fanconi syndrome: pathological and molecular characteristics compared with classical myelomaassociated Fanconi syndrome. Nephrol. Dial. Transplant. 25, 2982-2990 (2010).

61. Koo, H., Oh, D. H., Chun, Y. S. \& Kim, J. C. A case of crystalline keratopathy in monoclonal gammopathy of undetermined significance (MGUS). Korean J. Ophthalmol. 25, 202-205 (2011).

62. Dogan, S., Barnes, L. \& Cruz-Vetrano, W. P. Crystalstoring histiocytosis: report of a case, review of the literature (80 cases) and a proposed classification. Head Neck Pathol. 6, 111-120 (2012).

63. Kanagal-Shamanna, R. et al. Crystal-storing histiocytosis: a clinicopathological study of 13 cases. Histopathology 68, 482-491 (2016).

64. de Alba Campomanes, A. G. et al. Crystal-storing histiocytosis and crystalline keratopathy caused by monoclonal gammopathy of undetermined significance. Cornea 28, 1081-1084 (2009).

65. Gupta, V., El Ters, M., Kashani, K., Leung, N. \& Nasr, S. H. Crystalglobulin-induced nephropathy. J. Am. Soc. Nephrol. 26, 525-529 (2015).

66. Leung, N., Buadi, F., Song, K. W., Magil, A. B. $\&$ Cornell, L. D. A case of bilateral renal arterial thrombosis associated with cryocrystalglobulinaemia. Nephrol. Dial. Transplant. 3, 74-77 (2010).

67. Ball, N. J., Wickert, W., Marx, L. H. \& Thaell, J. F. Crystalglobulinemia syndrome. A manifestation of multiple myeloma. Cancer 71, 1231-1234 (1993)

68. Nasr, S. H. et al. Renal monoclonal immunoglobulin deposition disease: a report of 64 patients from a single institution. Clin. J. Am. Soc. Nephrol. 7 231-239 (2012)

69. Lin, J. et al. Renal monoclonal immunoglobulin deposition disease: the disease spectrum. J. Am. Soc. Nephrol. 12, 1482-1492 (2001).

70. Bridoux, F. et al. Unravelling the immunopathological mechanisms of heavy chain deposition disease with implications for clinical management. Kidney Int. 91 423-434 (2017)

71. Nasr, S. H. et al. Proliferative glomerulonephritis with monoclonal IgG deposits: a distinct entity mimicking immune-complex glomerulonephritis. Kidney Int. 65, 85-96 (2004)

72. Bhutani, G. et al. Hematologic characteristics of proliferative glomerulonephritides with nonorganized monoclonal immunoglobulin deposits. Mayo Clin. Proc. 90, 587-596 (2015).

73. Vignon, M. et al. The clinicopathologic characteristics of kidney diseases related to monotypic IgA deposits. Kidney Int. 91, 720-728 (2017).

74. Lloyd, I. E. et al. C3 glomerulopathy in adults: a distinct patient subset showing frequent association with monoclonal gammopathy and poor renal outcome. Clin. Kidney J. 9, 794-799 (2016).

75. Zand, L. et al. C3 glomerulonephritis associated with monoclonal gammopathy: a case series. Am. J. Kidney Dis. 62, 506-514 (2013)

76. Sethi, S. et al. Dense deposit disease associated with monoclonal gammopathy of undetermined significance. Am. J. Kidney Dis. 56, 977-982 (2010).

77. Kyle, R. A. et al. Prevalence of monoclonal gammopathy of undetermined significance. N. Engl. J. Med. 354, 1362-1369 (2006)

78. Larsen, C. P. et al. Membranoproliferative glomerulonephritis with masked monotypic immunoglobulin deposits. Kidney Int. 88, 867-873 (2015).

79. Lloyd, I. E. \& Khalighi, M. A. Glomerulonephritis with masked monotypic immunoglobulin deposits and concurrent lymphomatous infiltration. Am. J. Kidney Dis. 68, 640-644 (2016).

80. Yandrapalli, S., Gupta, S., Papanagnou, A., Karass, M. $\&$ Chugh, S. Thrombotic microangiopathy in renal transplanted patients: analysis of nationwide inpatien sample. Am. J. Kidney Dis. 69, A103 (2017).

81. Mahmood, U. et al. Monoclonal gammopathy of renal significance triggering atypical haemolytic uraemic syndrome. Nephrology 22, 15-17 (2017).

82. Blanc, C. et al. Anti-factor $\mathrm{H}$ autoantibodies in $\mathrm{C} 3$ glomerulopathies and in atypical hemolytic uremic syndrome: one target, two diseases. J. Immunol. 194 5129-5138 (2015).

83. Modesto-Segonds, A., Rey, J. P., Orfila, C., Huchard, G. $\&$ Suc, J. M. Renal involvement in POEMS syndrome. Clin. Nephrol. 43, 342-345 (1995).

84. Nakamoto, Y., Imai, H., Yasuda, T., Wakui, H. \& Miura, A. B. A spectrum of clinicopathological features of nephropathy associated with POEMS syndrome. Nephrol. Dial. Transplant. 14, 2370-2378 (1999).
85. Ye, W. et al. Renal impairment in patients with polyneuropathy, organomegaly, endocrinopathy, monoclonal gammopathy and skin changes syndrome: incidence, treatment and outcome. Nephrol. Dial. Transplant. 31, 275-283 (2016).

86. Said, S. M. et al. Myeloproliferative neoplasms cause glomerulopathy. Kidney Int. 80, 753-759 (2011).

87. Borza, D. B. et al. Recurrent Goodpasture's disease secondary to a monoclonal $\lg \mathrm{A}_{1}-\kappa$ antibody autoreactive with the $\alpha 1 / \alpha 2$ chains of type IV collagen. Am. J. Kidney Dis. 45, 397-406 (2005).

88. Coley, S. M., Shirazian, S., Radhakrishnan, J. $\&$ D'Agati, V. D. Monoclonal $\lg \mathrm{G}_{1} \kappa$ anti-glomerular basement membrane disease: a case report. Am. J. Kidney Dis. 65, 322-326 (2015).

89. Nasr, S. H. et al. The clinicopathologic characteristics and outcome of atypical anti-glomerular basement membrane nephritis. Kidney Int. 89, 897-908 (2016).

90. Debiec, H. et al. Recurrent membranous nephropathy in an allograft caused by $\operatorname{lgG}_{3} \mathrm{~K}$ targeting the PLA2 receptor. J. Am. Soc. Nephrol. 23, 1949-1954 (2012).

91. Best Rocha, A. \& Larsen, C. P. Membranous glomerulopathy with light chain-restricted deposits: a clinicopathological analysis of 28 cases. Kidney Int. Rep. 2, 1141-1148 (2017)

92. Dosa, S., Cairns, S. A., Mallick, N. P., Lawler, W. \& Williams, G. Relapsing Henoch-Schönlein syndrome with renal involvement in a patient with an IgA monoclonal gammopathy. A study of the results of immunosuppressant and cytotoxic therapy. Nephron 26, 145-148 (1980)

93. Zickerman, A. M. et al. IgA myeloma presenting as Henoch-Schönlein purpura with nephritis. Am. J. Kidney Dis. 36, E19 (2000).

94. Kyle, R. A. \& Gertz, M. A. Primary systemic amyloidosis: clinical and laboratory features in 474 cases. Semin. Hematol. 32, 45-59 (1995).

95. Randall, R. E., Williamson, W. C. Jr, Mullinax, F., Tung, M. Y. \& Still, W. J. Manifestations of systemic light chain deposition. Am. J. Med. 60, 293-299 (1976).

96. Landgren, O. et al. Risk of monoclonal gammopathy of undetermined significance (MGUS) and subsequent multiple myeloma among African American and white veterans in the United States. Blood 107, 904-906 (2006).

97. Delanaye, P., Glassock, R. J., Pottel, H. \& Rule, A. D. An age-calibrated definition of chronic kidney disease: rationale and benefits. Clin. Biochem. Rev. 37, 17-26 (2016).

98. Swaminathan, S. et al. Changing incidence of glomerular disease in Olmsted County, Minnesota: a 30-year renal biopsy study. Clin. J. Am. Soc. Nephrol. 1, 483-487 (2006).

99. Soares, S. M. et al. Bleeding complications after transcutaneous kidney biopsy in patients with systemic amyloidosis: single-center experience in 101 patients. Am. J. Kidney Dis. 52, 1079-1083 (2008).

100. Fish, R. et al. The incidence of major hemorrhagic complications after renal biopsies in patients with monoclonal gammopathies. Clin. J. Am. Soc. Nephrol. 5, 1977-1980 (2010).

101. Levi, I. M., Ben-Dov, I. Z., Klimov, A., Pizov, G. \& Bloom, A. I. Transjugular kidney biopsy: enabling safe tissue diagnosis in high risk patients. Isr. Med. Assoc. J. 13, 425-427 (2011).

102. Thompson, B. C. et al. Transjugular kidney biopsy. Am. J. Kidney Dis. 43, 651-662 (2004)

103. Huang, C. C. et al. IgG subclass staining in renal biopsies with membranous glomerulonephritis indicates subclass switch during disease progression. Mod. Pathol. 26, 799-805 (2013).

104. Bridoux, F. et al. Glomerulonephritis with isolated C3 deposits and monoclonal gammopathy: a fortuitous association? Clin. J. Am. Soc. Nephrol. 6, 2165-2174 (2011).

105. Herrera, G. A \& Turbat-Herrera, E. A Ultrastructural immunolabeling in the diagnosis of monoclonal lightand heavy-chain-related renal diseases. Ultrastruct. Pathol. 34, 161-173 (2010).

106. Herrera, G. A. The contributions of electron microscopy to the understanding and diagnosis of plasma cell dyscrasia-related renal lesions. Med. Electron. Microsc. 34, 1-18 (2001).

107. Herrera, G. A. Renal manifestations of plasma cell dyscrasias: an appraisal from the patients' bedside to the research laboratory. Ann. Diagn. Pathol. 4 174-200 (2000).

108. Vrana, J. A. et al. Classification of amyloidosis by laser microdissection and mass spectrometry-based proteomic analysis in clinical biopsy specimens. Blood 114, 4957-4959 (2009). 
109. Mohamed, N. \& Nasr, S. H. Renal amyloidosis. Surg. Pathol. Clin. 7, 409-425 (2014).

110. Royal, V. et al. IgD heavy-chain deposition disease: detection by laser microdissection and mass spectrometry. J. Am. Soc. Nephrol. 26, 784-790 (2015).

111. Leung, N., Barnidge, D. R. \& Hutchison, C. A Laboratory testing in monoclonal gammopathy of renal significance (MGRS). Clin. Chem. Lab. Med. 54 929-937 (2016)

112. Leung, N. et al. Urinary albumin excretion patterns of patients with cast nephropathy and other monoclonal gammopathy-related kidney diseases. Clin. J. Am. Soc. Nephrol. 7, 1964-1968 (2012).

113. Katzmann, J. A. et al. Screening panels for detection of monoclonal gammopathies. Clin. Chem. 55, 1517-1522 (2009).

114. Palladini, G. et al. A staging system for renal outcome and early markers of renal response to chemotherapy in AL amyloidosis. Blood 124, 2325-2332 (2014).

115. Palladini, G. et al. New criteria for response to treatment in immunoglobulin light chain amyloidosis based on free light chain measurement and cardiac biomarkers: impact on survival outcomes. J. Clin. Oncol. 30, 4541-4549 (2012).

116. Hutchison, C. A. et al. Serum free light chain measurement aids the diagnosis of myeloma in patients with severe renal failure. BMC Nephrol. 9, 11 (2008).

117. Kennard, A. et al. Comparison of Freelite and N Latex serum free light chain assays in subjects with end stage kidney disease on haemodialysis. Clin. Chem. Lab. Med. 54, 1045-1052 (2016).

118. Dispenzieri, A. et al. International Myeloma Working Group guidelines for serum-free light chain analysis in multiple myeloma and related disorders. Leukemia 23, 215-224 (2009).

119. Kumar, S. et al. International Myeloma Working Group consensus criteria for response and minimal residual disease assessment in multiple myeloma. Lancet Oncol. 17, e328-e346 (2016)

120. Treon, S. P. et al. MYD88 L265P somatic mutation in Waldenström's macroglobulinemia. N. Engl. J. Med. 367, 826-833 (2012).

121. Shanafelt, T. D., Ghia, P., Lanasa, M. C., Landgren, O. \& Rawstron, A. C. Monoclonal B cell lymphocytosis (MBL): biology, natural history and clinical management. Leukemia 24, 512-520 (2010).

122. Bochtler, T. et al. Gain of chromosome $1 \mathrm{q} 21$ is an independent adverse prognostic factor in light chain amyloidosis patients treated with melphalan/ dexamethasone. Amyloid 21, 9-17 (2014).

123. Bochtler, T. et al. Translocation $\mathrm{t}(11 ; 14)$ is associated with adverse outcome in patients with newly diagnosed $\mathrm{AL}$ amyloidosis when treated with bortezomib-based regimens. J. Clin. Oncol. 33, 1371-1378 (2015).

124. Muchtar, E. et al. Interphase fluorescence in situ hybridization in untreated $\mathrm{AL}$ amyloidosis has an independent prognostic impact by abnormality type and treatment category. Leukemia 31, 1562-1569 (2017).

125. Hillengass, J. et al. Whole-body computed tomography versus conventional skeletal survey in patients with multiple myeloma: a study of the International Myeloma Working Group. Blood Cancer J. 7, e599 (2017).

126. Chantry, A. et al. Guidelines for the use of imaging in the management of patients with myeloma. Br. J. Haematol. 178, 380-393 (2017).
127. Landgren, O. MRD testing in multiple myeloma: from a surrogate marker of clinical outcomes to an every-day clinical tool. Semin. Hematol. 55, 1-3 (2018).

128. Palladini, G. et al. Identification of amyloidogenic light chains requires the combination of serum-free light chain assay with immunofixation of serum and urine. Clin. Chem. 55, 499-504 (2009).

129. Wechalekar, A. D. et al. AL amyloidosis associated with IgM paraproteinemia: clinical profile and treatment outcome. Blood 112, 4009-4016 (2008).

130. Kourelis, T. V. et al. Outcomes of patients with renal monoclonal immunoglobulin deposition disease. Am. J. Hematol. 91, 1123-1128 (2016).

131. Ziogas, D. C. et al. Hematologic and renal improvement of monoclonal immunoglobulin deposition disease after treatment with bortezomib based regimens. Leuk. Lymphoma 58, 1832-1839 (2017).

132. Messiaen, T. et al. Adult Fanconi syndrome secondary to light chain gammopathy. Clinicopathologic heterogeneity and unusual features in 11 patients. Medicine (Baltimore) 79, 135-154 (2000).

133. Brouet, J. C., Clauvel, J. P., Danon, F., Klein, M $\&$ Seligmann, M. Biologic and clinical significance of cryoglobulins. A report of 86 cases. Am. J. Med. 57 775-788 (1974).

134. Bryce, A. H., Kyle, R. A., Dispenzieri, A. \& Gertz, M. A. Natural history and therapy of 66 patients with mixed cryoglobulinemia. Am. J. Hematol. 81, 511-518 (2006).

135. Neel, A. et al. Long-term outcome of monoclonal (type 1) cryoglobulinemia. Am. J. Hematol. 89 156-161 (2014)

136. Sidana, S. et al. Clinical presentation and outcomes of patients with type 1 monoclonal cryoglobulinemia. Am. J. Hematol. 92, 668-673 (2017).

137. Jones, D., Bhatia, V. K., Krausz, T. \& Pinkus, G. S. Crystal-storing histiocytosis: a disorder occurring in plasmacytic tumors expressing immunoglobulin $\mathrm{\kappa}$ ligh chain. Hum. Pathol. 30, 1441-1448 (1999).

138. Dotten, D. A., Pruzanski, W., Olin, J. \& Brown, T. C. Cryocrystalglobulinemia. Can. Med. Assoc. J. 114, 909-912 (1976)

139. Javaugue, V. et al. Long-term kidney disease outcomes in fibrillary glomerulonephritis: a case series of 27 patients. Am. J. Kidney Dis. 62, 679-690 (2013).

\section{Acknowledgements}

N.L. is supported by the generous donations of T. Kirshenbaum and J. Kirshenbaum as well as G. Kohler and A. Drennan. P.W.S. is supported by grants from the US Office of Research and Development, Medical Research Service, Department of Veterans Affairs (grant 1 IP1 BX001595), the US National Institutes of Health George M. O'Brien Kidney and Urological Research Centers Program (P30 DK079337) and the University of Alabama-Birmingham School of Medicine (AMC21 Multi-PI Grant and Anderson Innovation Award).

\section{Author contributions}

N.L., F.B., P.C., V.D.D., J.P.-F., J.D.G., G.A.H., A.J., D.J., E.K, R.A.K., H.J.L., C.P.L., G.S.M., G.M., M.M.P., V.R., P.W.S. and S.H.N. researched data for the article, contributed to discussions of its content, writing the manuscript, and review or editing of the manuscript before submission. V.B. researched data for the article and participated in review and/or editing of the manuscript before submission. A.C., F.C.F. and P.M.V. contributed to discussions of the article content and review and/or editing of the manuscript before submission. A.D.,
P.M. and B.M.W. contributed to discussions of the article content writing the manuscript and review or editing of the manuscript before submission. S.G. participated in review and/or editing of the manuscript before submission. V.K. contributed to writing the manuscript and review and/or editing of the manuscript before submission. H.J.L., V.S.R., S.S. and A.D.W. researched data for the article contributed to discussions of content and participated in review and/or editing of the manuscript before submission. C.P.V. contributed to discussions of the article content, writing the manuscript and review and/or editing of the manuscript before submission.

\section{Competing interests}

N.L. declares that he is a member of the advisory boards of BTC International, Prothena and Takeda; has received trial support from Omeros; and consults for Aduro. F.B. declares that he has received honoraria from Celgene and Janssen. P.C. declares that he is a member of the advisory board of The Binding Site, receives research support from The Binding Site and has received honoraria from Janssen. A.D. declares that she has received research grants from Alnylam, Celgene, GlaxoSmithKline, Janssen, Pfizer, Prothena and Takeda. F.C.F. declares that he has received an unrestricted grant from Genentech and Janssen and is a member of the advisory board for Alnylam. J.D.G. declares that he is a member of the advisory boards for Alnylam, Eidos and GlaxoSmithKline. V.K. declares that he has received honoraria from Amgen, Celgene and Takeda. H.J.L. declares that she has received research funding from Amgen and Takeda and is a member of the speaker's bureau for Amgen, Bristol-Myers Squibb, Celgene, Janssen and Takeda. H.L. declares that he has received research support from Amgen and Takeda and is a member of the speaker's bureau for Amgen, Bristol-Myers Squibb, Celgene, Janssen and Takeda and consults for PharmaMar. G.M. declares that he has received honoraria from Janssen, Pfizer and Prothena. P.M. declares that he is a member of the advisory boards of Amgen, Celgene and Janssen and has received clinical trial support from Janssen. S.H.N declares that he has received honoraria from Alnylam. C.P.V. declares that he has received honoraria from Amgen, Celgene, Janssen, Prothena and Takeda. P.M.V. declares that he is a member of the advisory boards of Bristol-Myers Squibb, Celgene, Janssen, Oncopeptides and TeneoBio and is a member of the speaker's bureau for Amgen and Janssen and consults for Celgene, Novartis, Oncopeptides and TeneoBio. B.M.W. declares that in October 2017 he became an employee of Janssen. All other authors declare no competing interests.

\section{Publisher's note}

Springer Nature remains neutral with regard to jurisdictional claims in published maps and institutional affiliations.

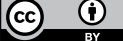

Open Access This article is licensed under a Creative Commons Attribution 4.0 International License, which permits use, sharing adaptation, distribution and reproduction in any medium or format, as long as you give appropriate credit to the original author(s) and the source, provide a link to the Creative Commons license, and indicate if changes were made. The images or other third party material in this article are included in the article's Creative Commons license, unless indicated otherwise in a credit line to the material. If material is not included in the article's Creative Commons license and your intended use is not permitted by statutory regulation or exceeds the permitted use, you will need to obtain permission directly from the copyright holder. To view a copy of this license, visit http://creativecommons.org/licenses/by/4.0/. 